Draft VERSION February 9, 2016

Preprint typeset using LTEX style emulateapj v. 5/2/11

\title{
FARGO3D: A NEW GPU-ORIENTED MHD CODE
}

\author{
Pablo Benítez-Llambay \\ Instituto de Astronomía Teórica y Experimental, Observatorio Astronónomico, Universidad Nacional de Córdoba. Laprida 854, X5000BGR, Córdoba, \\ Argentina
}

\author{
FRÉDÉRIC S. MASSET \\ Instituto de Ciencias Físicas, Universidad Nacional Autónoma de México (UNAM), Apdo. Postal 48-3,62251-Cuernavaca, Morelos, Mexico \\ Draft version February 9, 2016
}

\begin{abstract}
We present the FARGO3D code, recently publicly released. It is a magnetohydrodynamics code developed with special emphasis on protoplanetary disks physics and planet-disk interactions, and parallelized with MPI. The hydrodynamics algorithms are based on finite difference upwind, dimensionally split methods. The magnetohydrodynamics algorithms consist of the constrained transport method to preserve the divergence-free property of the magnetic field to machine accuracy, coupled to a method of characteristics for the evaluation of electromotive forces and Lorentz forces. Orbital advection is implemented, and an N-body solver is included to simulate planets or stars interacting with the gas. We present our implementation in detail and present a number of widely known tests for comparison purposes. One strength of FARGO3D is that it can run on both Graphical Processing Units (GPUs) or Central Processing unit (CPUs), achieving large speed up with respect to CPU cores. We describe our implementation choices, which allow a user with no prior knowledge of GPU programming to develop new routines for the CPU, and have them translated automatically for the GPU.
\end{abstract}

Subject headings: accretion, accretion disks - protoplanetary disks - hydrodynamics - methods: numerical

\section{INTRODUCTION}

Theories of planet-disk interactions have considerably evolved in the last two decades. A significant part of their progress is attributable to numerical experiments. In many of these, the role played by the different parameters of the problem has been deciphered by systematic, computationally expensive explorations of the parameter space. These could be achieved in part thanks to the steadily increasing power of computational resources, and in part by the development of fast algorithms adapted to the specifics of gas motion in thin, nearly Keplerian disks, known as orbital advection algorithms (Masset 2000; Johnson et al. 2008, Stone \& Gardiner 2010). Although two-dimensional (2D) calculations are still a valuable tool in tackling specific problems linked to planet-disk interactions, most of the latest results in this field have been obtained through three-dimensional calculations. In problems involving magnetic field tied to the matter, the prevalence of the magneto-rotational instability, which requires resolving the vertical dimension across the disk, implies that most studies of planet-disks interactions must be tackled through expensive, three dimensional calculations, with only a few notable exceptions.

The development of the CUDA language, aimed at tapping the huge computational resources of Graphical Processing Units (GPUs) for general purpose processing, commonly known as GPGPU computing, allows the development of astrophysical computational codes which can tackle expensive problems at relatively moderate cost. We can adopt as a rule of thumb that the same code runs between one and two orders of magnitude faster on a given high profile GPU than on a high profile CPU core of same generation. This ratio turns out to be of same order of magnitude as the vertical number of zones in many three-dimensional (3D) studies. To put it sim-

pbllambay@oac.unc.edu.ar

masset@ici.unam.mx ply, GPUs put 3D calculations at the cost of $2 \mathrm{D}$ calculations on a CPU core.

With this in mind, we have developed a new code that solves the equations of hydrodynamics (HD) or magnetohydrodynamics (MHD) on a mesh (either Cartesian, cylindrical or spherical) with special emphasis on describing protoplanetary disks and their interactions with forming planets.

While there is a plethora of astrophysical fluid dynamics codes, there are a lot fewer that can run on GPUs. One of them is the public Adaptive Mesh Refinement (AMR) code ENZO (Bryan et al. 2014), which contains hard coded CUDA versions of the PPM and MHD solvers. Like ENZO, the GAMER code (Schive et al. 2010) is another AMR code essentially oriented toward cosmological simulations, which contains a hydrodynamic solver coded in CUDA. Another one is the hydrodynamical code CHOLLA (Schneider \& Robertson 2015), which is based on high order Godunov's methods, and contains hard coded CUDA kernels. Another one is the PEnGUIn code (Fung et al. 2014), which is a Lagrangian, dimensionally split, shock capturing hydrocode that uses the Parabolic Piecewise Method (PPM, Colella \& Woodward 1984). It is written in CUDA and C. As memory is a significant concern in cosmological simulations, and since the standard RAM available on a cluster node is generally much larger than the RAM on board of the GPUs, ENZO and GAMER constantly transfer back and forth data between the CPU and GPU to benefit at the same time from the large memory of the host (CPU) and the large computational throughput of the GPU. Since these transfers constitute a bottleneck for effective GPU performance, the GAMER code uses sophisticated asynchronous data transfers in order to hide their latency. Our needs are very different: in the domain of predilection of FARGO3D, that of protoplanetary disks and their interactions with embedded planets, speed is generally much more a concern than memory. We therefore require that our 
whole simulation fits on the memory of the GPU(s), in order to avoid data transfer. All our routines, including those dedicated to boundary conditions, run on the GPU. Consequently, we have striven to obtain the smallest possible memory footprint per cell.

When the HD or MHD algorithms are directly coded in CUDA, a user who wishes to amend the core routines, or who wishes to incorporate new physics into them must be proficient in CUDA. We have adopted a radically different approach: we have developed all our code for CPU, and enforced the use of strict syntax rules in all computationally expensive routines which expose their parallelism, so that they can be translated automatically during compilation to CUDA code. This allows users who have no prior knowledge of GPU programming to easily modify the code for their own needs. This also made the development of the code much faster and reliable. The scope of this paper is a comprehensive description of the algorithms used in our code; we do not present the syntax rules that we have developed to enable the automatic translation, which can be found in the online manual. We nonetheless describe the conceptual ideas of this process in section 4.2

The code that we have developed is based on ideas similar to those used in the ZEUS code (Stone \& Norman 1992a). In this code, the velocities are not centered on the cells but staggered on their faces. This allows us to calculate easily the fluxes of mass, momenta and specific energy on the cell edges, by the use of upwind methods.

ZEUS-like codes have oftentimes been referred to as finite difference codes, as opposed to the so-called finite volume codes. These, like those based on Godunov's method that have emerged in astrophysics in the past two decades, in which Riemann's problem is used to evaluate fluxes at the faces between cells, automatically ensure the conservation of the physical quantities for which a flux can be evaluated. This could suggest that finite difference codes like the one we present in this work do not have such conservation properties. In fact, a significant fraction of the sub steps performed over a full update correspond to finite volume operations. In particular, we shall see that FARGO3D conserves mass and momenta to machine accuracy.

We summarize hereafter the salient points of our implementation choices:

1. Our method is dimensionally split, thereby requiring as few as possible temporary arrays. Our code has probably the smallest possible memory imprint, which is well suited to GPUs, which have moderate amounts of random access memory (RAM) compared to CPUs.

2. Staggered mesh codes, contrary to Godunov's methods, do not have issues with steady flows with sources terms. This bears some importance in many situations in protoplanetary disks, for which the vertical and rotational equilibrium of the unperturbed disk, as well as that of the envelope that appears around embedded planets, must be accurately captured by the method.

3. The cost of a full time step with a staggered mesh code is significantly smaller than that of a Godunov's method.

4. Codes based on Godunov's method use generally fluxes of total energy, thus enforcing the conservation of energy to machine accuracy. On the other hand, staggered mesh codes consider the fluxes of internal energy, and do not fulfill the conservation of energy to machine accuracy. Although this at first sight may seem a disadvantage, it is not so: in protoplanetary disks, which usually have a large Mach number, the kinetic energy is two to three orders of magnitude larger than the internal energy. Any truncation error affecting the kinetic energy is forcibly transferred to the internal energy budget, which compounds the relative error. This problem is known as the high Mach number problem (Ryu et al. 1993, Trac \& Pen 2004). Many planet-disk interaction problems require an accurate advection of entropy in the planet's coorbital region. It is therefore desirable to deal with the internal energy separately.

5. Inclusion of new processes generally requires significantly more work with Godunov's method, such as rewriting or significantly editing the Riemann solver, whereas it is easier to incorporate new or different physics in staggered mesh codes through the operator splitting technique. Although we have striven to make the process of translation to GPUs as transparent and automatic as possible, the use of simple routines ensures that our code can be straightforwardly adapted to the user's needs, without requiring proficiency in GPU programming.

6. The predecessor of our new code, FARGO, has been intensively used in the field of planet-disk interactions, and proven to perform well over the whole range of planetary masses, from deeply embedded, low mass objects, which require finely tuned rotational equilibrium and accurate entropy and vortensity advection, to giant planets, which clear a gap and excite strong shocks in their immediate vicinity. Although Godunov's codes are better at handling shocks than staggered mesh codes, the latter have been found to yield similar results to the former in problems involving gap clearing and shocks excitation (de Val-Borro et al. 2006).

As does its predecessor, the FARGO3D code includes orbital advection. Initially, FARGO (Fast Advection in Rotating Gaseous Objects) was an orbital advection algorithm (Masset 2000). This acronym was then used to name the code originally based on this algorithm and publicly released in 2005. The orbital advection has been extended to MHD in the present implementation, using the upstream averaged electric field method of Stone \& Gardiner (2010).

One of the main novelties introduced in FARGO3D is that it can be run effortlessly on GPU platforms with a high computational throughput. This, together with the fact that it features orbital advection in its HD and MHD versions, makes it a tool of choice to study protoplanetary disk dynamics and planetdisk interactions, which are extremely demanding in terms of computational power.

This paper is organized as follows: in section 2 we present the main characteristics of the code, list the equations that it solves and introduce our notation. In section 3 we present a flow chart of the code and describe step-by-step the numerical methods used to solve the governing equations. In section 4 we discuss some implementation details, in particular our choice to export computationally expensive routines to the GPU. In section 5 we present a set of tests on different problems in HD and MHD. In section 6, we provide some 
discussion on the impact of orbital advection on the properties of the code, both in HD and MHD, and we also present potential pitfalls of single precision calculations in the context of astrophysical disks. Finally, we discuss ongoing and future developments in section 7 Throughout the manuscript the reader will find many superscripts referring to end notes. These refer to the specifics of our implementation and should significantly speed up the learning process of the reader interested in modifying the code.

\section{OVERVIEW}

\subsection{Governing equations}

FARGO3D solves the equations of HD or MHD on an Eulerian mesh, which can be either Cartesian, cylindrical or spherical. As its name indicates, it is designed to solve threedimensional problems, but it can also be used in lower dimension (one or two-dimensions). The continuity equation is:

$$
\frac{\partial \rho}{\partial t}+\nabla \cdot(\rho \vec{v})=0,
$$

where $\rho$ is the volumic density, and $\vec{v}$ is the velocity of the fluid with respect to the mesh. The Navier-Stokes equation reads:

$$
\begin{aligned}
\rho\left(\frac{\partial \vec{v}}{\partial t}+\vec{v} \cdot \nabla \vec{v}\right)= & -\nabla P+\frac{1}{\mu_{0}}(\nabla \times \vec{B}) \times \vec{B}+\nabla \cdot \vec{T}+\overrightarrow{F_{e x t}} \\
& -[2 \vec{\Omega} \times \vec{v}+\vec{\Omega} \times(\vec{\Omega} \times \vec{r})+\dot{\vec{\Omega}} \times \vec{r}] \rho,
\end{aligned}
$$

where $P$ is the pressure, $\vec{B}$ the magnetic field, and $\vec{F}_{\text {ext }}$ is any external force (e.g. gravity force), and where the second term of the right hand side is dealt with only if MHD is included. The last term of Eq. (2) accounts for a possible rotation of the mesh about the vertical axis, at a rate $\vec{\Omega}$ which can vary with time. The third term features the stress tensor $\vec{T}$, which has the following expression:

$$
\vec{T}=\rho v\left[\nabla \vec{v}+(\nabla \vec{v})^{T}-\frac{2}{3}(\nabla \cdot \vec{v}) \vec{I}\right],
$$

where $v$ is the kinematic viscosity and $\vec{I}$ is the unit tensor of same rank as the tensor $\nabla \vec{v}$. For the energy equation, we use as discussed in section 1 a non-conservative form using the volumic internal energy $e$ :

$$
\partial_{t} e+\nabla \cdot(e \vec{v})=-P \nabla \cdot \vec{v} .
$$

Finally, when MHD is included, we solve the induction equation, which reads:

$$
\frac{\partial \vec{B}}{\partial t}=\nabla \times(\vec{v} \times \vec{B}-\eta \nabla \times \vec{B}),
$$

where $\eta$ is the Ohmic diffusivity. The Eqs. (1) to (5) are closed using an equation of state, giving a relation between pressure or the internal energy. In the public release ${ }^{1}$, two forms for the equation of state are provided:

$$
P=c_{s}^{2} \rho,
$$

and

$$
P=(\gamma-1) e,
$$

where respectively $c_{s}$ is the isothermal sound speed and $\gamma$ the ratio of specific heats at constant pressure and volume. The first form given by Eq. (6) is often called (locally) isothermal

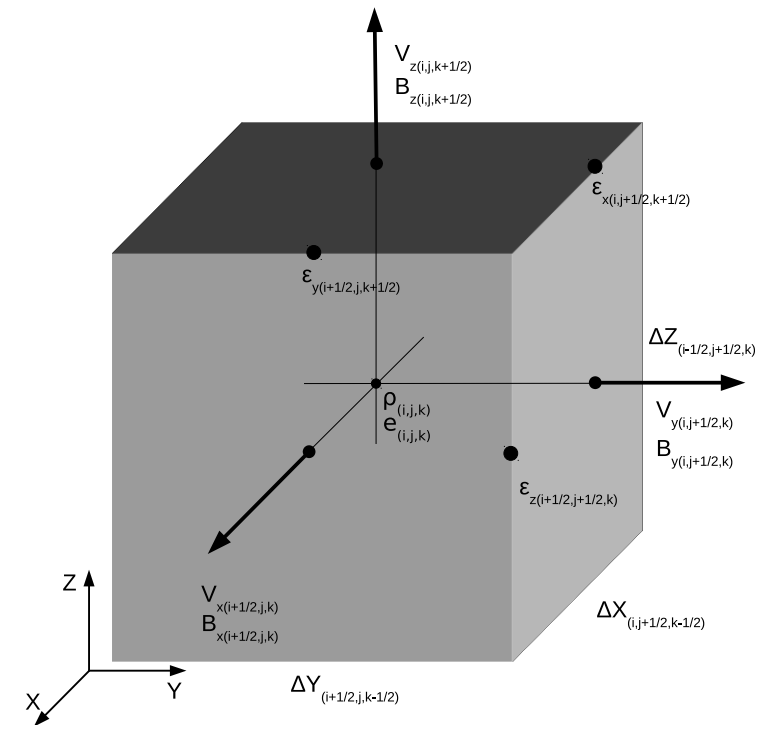

FIG. 1. - Sketch of a cell in FARGO3D. Vectorial quantities $\left(v_{x}, v_{y} v_{z}, B_{x}\right.$, $\left.B_{y} B_{z}\right)$ are located on the faces, while scalar ones $(e, \rho)$ are cell centered. Electromotive forces $\varepsilon$ are defined at middle of the edges (see section 3.6). Also shown are the edge lengths $(\Delta X, \Delta Y, \Delta Z)$, which generally depend on $j$ and $k$.

equation of state. Its field $c_{s}(\vec{r})$ is constant in time and given by the initial conditions. When this equation is used, Eq. (4) is decoupled from the others and does not have to be solved. The second form, often called adiabatic or ideal equation of state, is the relation between the internal energy and pressure. This form is commonly used when Eq. (4) has to be solved.

\subsection{Coordinate names}

The three different directions are generically named $X, Y$ and $Z$ in our implementation, even if the mesh geometry is non-Cartesian. Tab. 1 lists the correspondence between these names and the corresponding coordinate in the different geometries.

In Cartesian coordinates one may use the shearing sheet ${ }^{2}$ formalism to describe a sheared flow in a rotating frame, subject to the standard expansion of the effective (centrifugal plus central) potential. This will trigger the use of a different momentum component along the $X$ direction (see Tab. 2) and the inclusion of specific source terms (see section 3.3). The frame rotating rate is not allowed to vary in the shearing sheet case ${ }^{3}$.

\subsection{Centering and notation}

We store the face-centered values of vectorial quantities (velocity and magnetic field components) while we store the cell-centered values of scalar quantities (internal energy and density).

In figure 1 we display a sketch of a single cell, showing the centering of different quantities. We adopt half integer indices for edges, while integer indices correspond to cell centers. The rule we follow is: if a quantity $Q$ is defined at the center of cell $i, j, k$, its value there reads $Q_{i, j, k}$, while if it is defined at the center of the interface between cell $i, j, k$ and cell $i, j+1, k$, its value reads $Q_{i, j+\frac{1}{2}, k}$.

\section{METHODS}

FARGO3D solves the hydrodynamical equations with a time-explicit method, using operator splitting and upwind techniques on an Eulerian mesh. Some differential equations 


\begin{tabular}{l||ccc} 
Geometry & $X$ & $Y$ & $Z$ \\
\hline Shearing sheet & along advection & along gradient of unperturbed velocity & perpendicular to the previous ones \\
Cylindrical & azimuth $(\phi)$ & radius $(r)$ & $Z$ \\
Spherical & azimuth $(\phi)$ & radius $(r)$ & colatitude $(\theta)$
\end{tabular}

TABLE 1

CORRESPONDENCE BETWEEN NAMES $X, Y, Z$ AND THE COORDINATE NAME IN DIFFERENT GEOMETRIES.

are discretized as finite differences, whereas others are solved using finite volume methods. To update the magnetic field with the induction equation 5, we use the Method of Characteristics (MOC, Stone \& Norman 1992b) and to preserve the free divergence property of the magnetic field we use the constrained transport method (CT, Evans \& Hawley 1988).

Fig. 2 shows a flow chart corresponding to a complete time step (or full update). The governing equations are solved by performing in succession the different substeps shown in this figure. In the following two subsections we explain how we split the equations of HD into a source and transport step, corresponding to boxes 7 and 12 in Fig. 2. The practical application of these steps will be discussed in further detail in Section 3.3 and 3.4. Our implementation of the CFL condition is presented in Section 3.5 Additional terms required by MHD are discussed in section 3.6, and the FARGO algorithm for MHD is discussed in Section 3.7. We conclude the section with a discussion of the orbital integrator for planets, in Section 3.8 .

\subsection{Operator splitting technique}

As is done frequently in (magneto)hydrodynamical solvers, the update of the different fields is done in a step-by-step manner, along the lines of the operator splitting technique (e.g. Stone \& Norman 1992a). Consider a problem defined by:

$$
\frac{\partial U}{\partial t}+\mathcal{A}(U)=0, \quad U(0)=U_{0} .
$$

If we can decompose $\mathcal{A}$ such that $\mathcal{A}=\mathcal{A}_{1}+\mathcal{A}_{2}$, the solution of Eq. (8) could be obtained as the linear combination of the solutions of:

$$
\begin{aligned}
& \frac{\partial U_{1}}{\partial t}+\mathcal{A}_{1}\left(U_{1}\right)=0, \\
& \frac{\partial U_{2}}{\partial t}+\mathcal{A}_{2}\left(U_{2}\right)=0 .
\end{aligned}
$$

In a finite differences scheme, the approximate solution can be written in the form:

$$
\begin{aligned}
& \frac{U^{1}-U^{0}}{\Delta t}=-A_{1}\left(U^{0}\right), \\
& \frac{U^{2}-U^{1}}{\Delta t}=-A_{2}\left(U^{1}\right) .
\end{aligned}
$$

We note that in hydrodynamics, differential equations have the general form 1 .

$$
\frac{\partial Q}{\partial t}+\nabla \cdot(Q \vec{v})=\mathcal{S}(Q, \vec{v}, t)
$$

where $Q$ can be any quantity (such as a momentum component), and $\mathcal{S}$ are source or sink terms of $Q$ (for instance a body force). In that case, splitting Eq. (13) is straightforward:

$$
\begin{aligned}
& A_{1}=-\mathcal{S} \\
& A_{2}=\nabla \cdot(Q \vec{v})
\end{aligned}
$$

\footnotetext{
${ }^{1}$ If $Q$ is a vectorial quantity, Eq. (13) is still valid component wise.
}

The technique of operator splitting therefore divides the problem into two partial ones, the Source Step and the Transport Step (Stone \& Norman 1992a). The equations corresponding to the partial problems read respectively:

$$
\partial_{t} Q=\mathcal{S}(Q, \vec{v}, t)
$$

and

$$
\partial_{t} Q+\nabla \cdot(Q \vec{v})=0
$$

A complete time step is therefore composed of a partial update from $Q\left(t_{0}\right)=Q^{0} \rightarrow Q^{1}$ by the source step described by Eq. (16), and a subsequent update from $Q^{1} \rightarrow Q^{2}=Q\left(t_{0}+\Delta t\right)$ by the transport step of Eq. (17). In addition, a substep dedicated to the interaction of the flow with the magnetic field may be added, and will be considered in section 3.6

\subsection{Splitting source and transport in practice}

We entertain here the equations of HD in spherical coordinates, the frame being in rotation about the polar axis with an angular velocity $\Omega_{f}(t)$. The reason for the possible time dependence of the frame rotation rate is that in many circumstances it is useful that the $x$ axis (direction of azimuth's origin) tracks either a planet or its guiding-center, which yields a non constant rotation rate if the planet is on a non-circular orbit in the first case, or if it is migrating in the second case, or if the orbit is inclined in either case. The cases of Cartesian and cylindrical coordinates can be straightforwardly worked out, and are summed up at the end of this section.

The equations that govern the flow are the continuity equation (Eq. 1), where the divergence operator, in spherical coordinates, is:

$$
\nabla . \vec{u} \equiv \frac{1}{r^{2}} \partial_{r}\left(r^{2} u_{r}\right)+\frac{1}{r \sin \theta} \partial_{\phi} u_{\phi}+\frac{1}{r \sin \theta} \partial_{\theta}\left(u_{\theta} \sin \theta\right),
$$

the Euler equation (Eq. 2), which can be recast respectively in radius, azimuth and colatitude as (the color coding of the terms will be explained later):

$$
\begin{gathered}
D_{t} v_{r}=\frac{v_{\theta}^{2}+\left(v_{\phi}+r \sin \theta \Omega_{f}\right)^{2}}{r}-\frac{\partial_{r} P}{\rho}-\partial_{r} \Phi, \\
D_{t} v_{\phi}=-r \sin \theta \dot{\Omega}_{f}-\frac{v_{r} v_{\phi}+v_{\theta} v_{\phi} \cot \theta}{r} \\
-2 \Omega_{f}\left(v_{r} \sin \theta+v_{\theta} \cos \theta\right)-\frac{\partial_{\phi} P}{r \sin \theta \rho}-\frac{\partial_{\phi} \Phi}{r \sin \theta}
\end{gathered}
$$

and

$$
D_{t} v_{\theta}=-\frac{v_{r} v_{\theta}}{r}+\frac{\left(v_{\phi}+r \sin \theta \Omega_{f}\right)^{2}}{r} \cot \theta-\frac{\partial_{\theta} P}{r \rho}-\frac{\partial_{\theta} \Phi}{r},
$$

where the Lagrangian derivative $D_{t}$ is defined by:

$$
D_{t} Q=\partial_{t} Q+v_{r} \partial_{r} Q+\frac{v_{\phi}}{r \sin \theta} \partial_{\phi} Q+\frac{v_{\theta}}{r} \partial_{\theta} Q
$$

for any scalar quantity $Q$, and the energy equation (4):

$$
\partial_{t} e+\nabla \cdot(e \vec{v})=-P \nabla \cdot \vec{v} .
$$




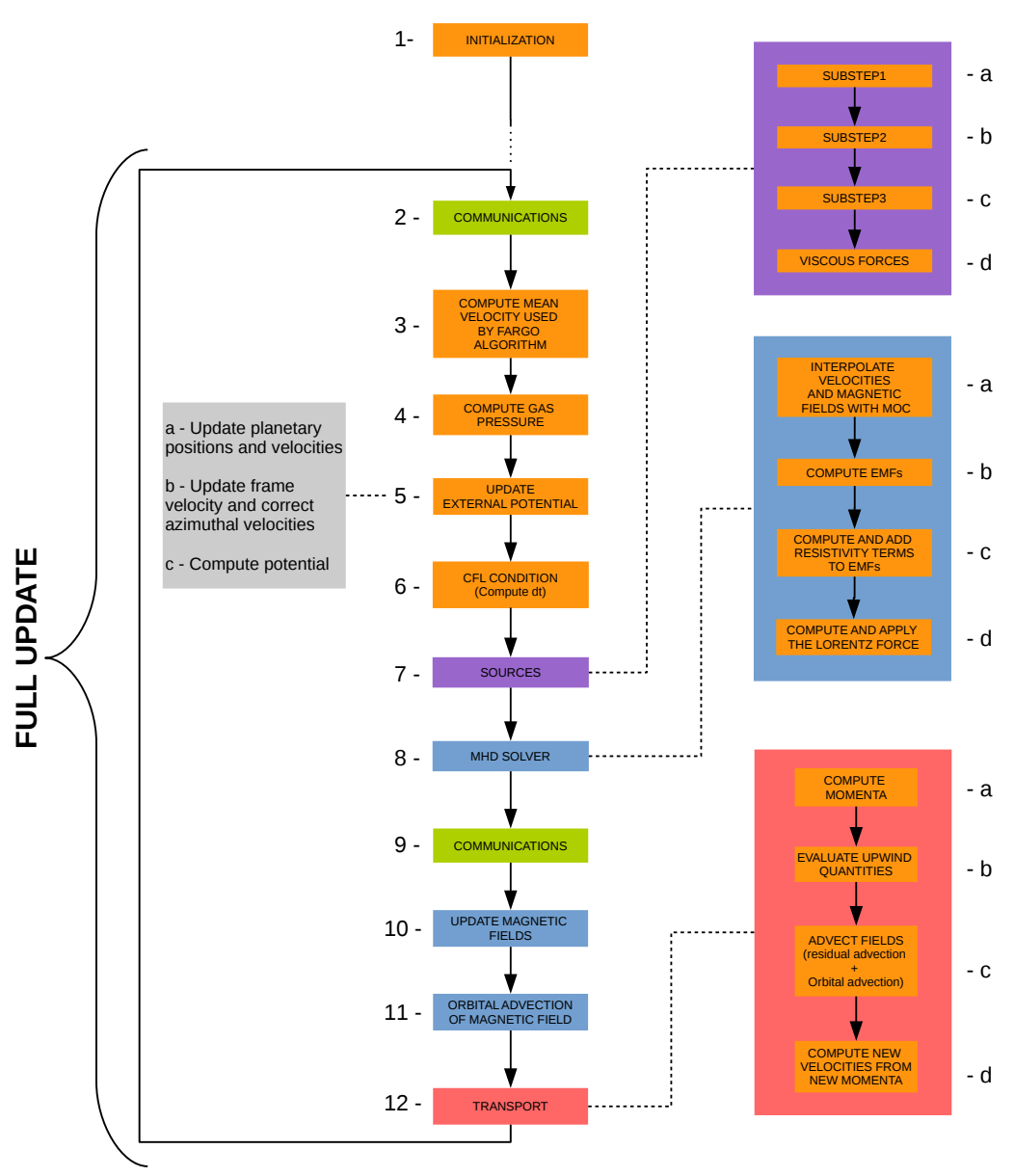

FIG. 2.- Flow chart of the operations performed in succession in FARGO3D during a full update. Boxes with blue background are specific to the MHD case. The corresponding substeps are run only when the code is compiled for MHD. Communications (cells 2 and 9) are discussed in section 4.1 The evaluation of the mean velocity at cell 3 is discussed in sections 3.5.1 and section 3.5.2 The evaluation of the gas pressure at cell 4 is done either according to Eq. (6) or according to (7). Calculations relative to the external potential (cell 5) are discussed in section 3.8 The time step evaluation of cell 6 is detailed in section 3.5 The detail of the different source steps of box 7 are given in section 3.3 and in appendix B for the viscous forces. Algorithms specific to MHD, corresponding to boxes 8 , 10 and 11, are described in sections 3.6 Finally, algorithms specific to the transport step (box 12) are described in section 3.4

Using Eqs. (1) and (19), we can write:

$$
\partial_{t}\left(\rho v_{r}\right)+\nabla \cdot\left(\rho v_{r} \vec{v}\right)=\rho \frac{v_{\theta}^{2}+\left(v_{\phi}^{t}\right)^{2}}{r}-\partial_{r} P-\rho \partial_{r} \Phi
$$

where, for the sake of brevity, we use $v_{\phi}^{t}=v_{\phi}+r \sin \theta \Omega_{f}$, the azimuthal velocity in the inertial frame. Similarly, using the specific angular momentum $j=r \sin \theta v_{\phi}+r^{2} \sin ^{2} \theta \Omega_{f}$, one may recast Eq. 201 as:

$$
\partial_{t}(\rho j)+\nabla \cdot(\rho j \vec{v})=\rho D_{t} j=-\partial_{\phi} P-\rho \partial_{\phi} \Phi .
$$

Finally, using Eqs (1) and (21), we can write:

$$
\partial_{t}\left(\rho r v_{\theta}\right)+\nabla \cdot\left(\rho r v_{\theta} \vec{v}\right)=\rho\left(v_{\phi}^{t}\right)^{2} \cot \theta+\partial_{\theta} P+\rho \partial_{\theta} \Phi .
$$

This equation on the "meridional momentum" has also been considered by Kley et al. (2009) in their implementation of the NIRVANA code. As we have seen in section 3.1, a transport substep is a combination of routines that solves Eq. (17) on the mesh, for an arbitrary quantity $Q$. We see from the above that, by transporting respectively the variables $\rho, \rho v_{r}$, $\rho j, \rho r v_{\theta}$ and $e$ (when we want to solve the energy equation), we take into account all the terms in black in Eqs. (19), 20) and (21). The terms in blue, which also appear on the right hand side of Eqs. (24), (25) and 26) (scaled by $\rho$ and geometric factors), are dealt with outside of the transport steps, in the source substep, which is the subject of the next section. The name source step is somewhat misleading as many source terms of Eqs. (19), 20) and 21) are dealt with during the conservative update of the transport step. In particular, the Coriolis force and all source terms involving a product of different velocity components are embedded in the transport step. This is of particular importance in the context of planets in disks. Kley (1998) has shown, by considering a gap-opening planet in a disk, that failing to deal with Coriolis forces in a conservative manner can lead to incorrect gap surface density profiles. Tab. 2 generalizes the above discussion to Cartesian and cylindrical geometries, and shows the quantities to transport in each case. The Cartesian case is trivial and does not allow for a rotation of the frame about the vertical axis.

\subsection{Source step}

In the source step, described by Eq. (16), all terms that are not included in the transport step must be considered. Specifying to the case of spherical geometry contemplated in the previous section, these are the terms that appear in 


\begin{tabular}{l||ccc} 
Geometry & X-momentum & Y-momentum & Z-momentum \\
\hline Cartesian & $\rho v_{x}$ & $\rho v_{y}$ & $\rho v_{z}$ \\
Shearing sheet & $\rho\left(v_{x}+2 \Omega_{f} y\right)$ & $\rho v_{y}$ & $\rho v_{z}$ \\
Cylindrical & $\rho\left(r v_{\phi}+r^{2} \Omega_{f}\right)$ & $\rho v_{r}$ & $\rho v_{z}$ \\
Spherical & $\rho\left(r v_{\phi} \sin \theta+r^{2} \sin ^{2} \theta \Omega_{f}\right)$ & $\rho v_{r}$ & $\rho r v_{\theta}$
\end{tabular}

TABLE 2

QUANTITIES TRANSPORTED IN $X, Y$ AND $Z$ TO ENSURE A FORMULATION AS CONSERVATIVE AS POSSIBLE, FOR THE DIFFERENT GeOMETRIES IMPLEMENTED. NOTE THAT FARGO3D'S NOTATION DIFFERS FROM THE USUAL NOTATION FOR THE SHEARING SHEET BY A SWAP OF THE $X$ AND $Y$ COORDINATES.

blue in Eqs (19), (20), (21) and (23) if the energy equation is solved. They correspond respectively to the centrifugal force, the pressure gradient, the body forces and the work done by pressure forces. All other terms in Eqs. (19), 20) and (21) are included in the conservative update of the transport step.

In addition, as we shall see in section 3.6, additional source terms arise from the Lorentz force in the MHD case.

We follow the procedure of Stone \& Norman (1992a), separating the source step into three sub-steps:

- Sub-step 1: we update ${ }^{4}$ the velocity field by pressure gradients and gravitational forces. This corresponds to the terms in blue in Eqs. (19) to 21), and to the cell 7a of the flow chart of Fig. 2

- Sub-step 2: we add an artificial von NeumannRichtmyer viscosity and corresponding heating terms ${ }^{5}$, in the exact same manner as described by Stone \& Norman (1992a). Instead of using a covariant form in curvilinear coordinates, component-wise expressions are employed in all cases. This corresponds to the cell $7 \mathrm{~b}$ of the flow chart of Fig. 2 .

- Sub-step 3: we add the work done by pressure forces ${ }^{6}$, corresponding to the blue term of Eq. (23), if the energy equation is solved. This corresponds to cell 7c of Fig.2

Below we show the source terms that have to be included in Sub-step 1 for different geometries:

\section{Cartesian case:}

$$
\frac{\partial v_{\chi}}{\partial_{t}}=-\frac{\partial_{\chi} P}{\rho}-\partial_{\chi} \Phi,
$$

where $\chi$ is an arbitrary direction.

\section{Shearing sheet:}

$$
\begin{aligned}
& \frac{\partial v_{x}}{\partial_{t}}=-\frac{\partial_{x} P}{\rho}-\partial_{x} \Phi \\
& \frac{\partial v_{y}}{\partial_{t}}=-\frac{\partial_{y} P}{\rho}-\partial_{y} \Phi+2 \Omega_{f} v_{x}-4 A \Omega_{f} y \\
& \frac{\partial v_{z}}{\partial_{t}}=-\frac{\partial_{z} P}{\rho}-\partial_{z} \Phi
\end{aligned}
$$

\section{Cylindrical case:}

$$
\begin{aligned}
& \frac{\partial v_{\phi}}{\partial t}=-\frac{1}{r} \frac{\partial_{\phi} P}{\rho}-\frac{1}{r} \partial_{\phi} \Phi \\
& \frac{\partial v_{r}}{\partial t}=-\frac{\partial_{r} P}{\rho}-\partial_{r} \Phi+\frac{\left(v_{\phi}^{t}\right)^{2}}{r} \\
& \frac{\partial v_{z}}{\partial t}=-\frac{\partial_{z} P}{\rho}-\partial_{z} \Phi
\end{aligned}
$$

\section{Spherical case:}

$$
\begin{aligned}
& \frac{\partial v_{\phi}}{\partial t}=-\frac{1}{r \sin \theta} \frac{\partial_{\phi} P}{\rho}-\frac{1}{r \sin \theta} \partial_{\phi} \Phi \\
& \frac{\partial v_{r}}{\partial t}=-\frac{\partial_{r} P}{\rho}-\partial_{r} \Phi+\frac{\left(v_{\phi}^{t}\right)^{2}+v_{\theta}^{2}}{r} \\
& \frac{\partial v_{\theta}}{\partial t}=-\frac{1}{r} \frac{\partial_{\theta} P}{\rho}-\frac{1}{r} \partial_{\theta} \Phi+\frac{\left(v_{\phi}^{t}\right)^{2} \cot \theta}{r}
\end{aligned}
$$

These source terms are applied directly to the velocity components. The staggering of the velocity fields makes trivial the evaluation of the pressure and potential gradients. Geometrical source terms (centrifugal forces) require in general averaging the information of several cells. In such cases we take the arithmetic average of the velocities, then we evaluate the square, as shown in the following example for the radial velocity in cylindrical geometry:

$$
\begin{aligned}
& \frac{v_{r i j-\frac{1}{2} k}^{b}-v_{r i j-\frac{1}{2} k}^{a}}{\Delta t}= \\
& \frac{1}{r}\left[\frac{1}{4}\left(v_{\phi_{i-\frac{1}{2} j k}}^{t}+v_{\phi_{i+\frac{1}{2} j k}}^{t}+v_{\phi_{i-\frac{1}{2} j-1 k}^{t}}^{t}+v_{\phi_{i+\frac{1}{2} j-1 k}}^{t}\right)\right]^{2},
\end{aligned}
$$

where the superscripts $a$ and $b$ denote the intermediate values at the beginning and end of the substep, respectively. Generalization to other geometries or directions is straightforward. The source term of Eq. (23) is applied as described by Eq. (40) of Stone \& Norman (1992a), valid for gamma-law gases.

The implementation of the viscous stress tensor is presented in appendix $B$

\subsection{Transport step}

As we have seen in section 3.1, the transport step consists in solving Eq. (17), which reads as a conservation law for the quantity $Q$ ( $Q$ being any of the quantities in the list given in section 3.2]) The integral form of Eq. (17) is, using the divergence theorem:

$$
\frac{\partial}{\partial t} \iiint_{V} Q d V+\iint_{\partial V} Q \vec{v} \cdot d \vec{S}=0,
$$

where we have assumed that the control volume $V$ has no explicit temporal dependency. Thus, time variations of $Q$ inside a control volume are due exclusively to its flux across the boundary $\partial V$ of the control volume. If $Q$ is defined at a cell center, the finite difference representation of Eq. (39) is:

$$
\begin{aligned}
\frac{Q_{i j k}^{n+1}-Q_{i j k}^{n}}{\Delta t} \mathcal{V}= & -\left[\mathcal{F}_{X i+\frac{1}{2} j k}-\mathcal{F}_{X i-1 / 2 j k}\right. \\
& +\mathcal{F}_{Y i j+1 / 2 k}-\mathcal{F}_{Y i j-1 / 2 k} \\
& \left.+\mathcal{F}_{Z i j k+1 / 2}-\mathcal{F}_{Z i j k-1 / 2}\right]^{n+1 / 2},
\end{aligned}
$$


where $\mathcal{V}$ is the volume of the cell and $\mathcal{F}$ is the flux of $Q$ across the faces of the cubic cell. Expressions of the fluxes along other directions can be straightforwardly inferred from Eq. (45). For multidimensional advection, we reduce the transport problem to several one-dimensional problems, each one updating partially the field with the corresponding fluxes:

$$
\begin{aligned}
& {\left[Q_{i j k}^{n+a}-Q_{i j k}^{n}\right] \mathcal{V}=-\Delta t\left[\mathcal{F}_{X i+1 / 2 j k}-\mathcal{F}_{X i-1 / 2 j k}\right]^{n+1 / 2}} \\
& {\left[Q_{i j k}^{n+b}-Q_{i j k}^{n+a}\right] \mathcal{V}=-\Delta t\left[\mathcal{F}_{Y i j+1 / 2 k}-\mathcal{F}_{Y i j-1 / 2 k}\right]^{n+1 / 2}} \\
& {\left[Q_{i j k}^{n+1}-Q_{i j k}^{n+b}\right] \mathcal{V}=-\Delta t\left[\mathcal{F}_{Z i j k+1 / 2}-\mathcal{F}_{Z i j k-1 / 2}\right]^{n+1 / 2}}
\end{aligned}
$$

Note that the fluxes in Eq. (42) are evaluated using the quantity $Q$ at the intermediate stage $n+a$, and similarly in Eq. (43), at the intermediate stage $n+b$, thus in principle the solution depends on the order of evaluation, which is a characteristic of numerical schemes based on dimensionally split methods (Stone \& Norman 1992a). Performing in succession the operations described by Eqs. (41) to (43) amounts to take into account all terms in black in the right hand side of Eqs. (19) to 21) (except for the term in $\dot{\Omega}_{f}$ ). These operations correspond to cell 12c of Fig. 2

We note that even though Eq. (25) embeds the first term of the right hand side of Eq. (20), the update of the frame angular velocity (first part of cell $5 \mathrm{~b}$ of Fig. 2 ) is not performed between the beginning and the end of the transport substep (respectively cells 12a and $12 \mathrm{~d}$ of Fig. 22, so that the new velocities do not reflect the variation of the frame rotation rate. Instead, upon the update of the frame angular velocity, we must explicitly correct the azimuthal velocities ${ }^{7}$. In the spherical case, this is done as follows:

$$
v_{\phi_{i j k}}^{c}=v_{\phi_{i j k}}^{b}-\left(\Omega_{f}^{\prime}-\Omega_{f}\right) r \sin \theta,
$$

where $\Omega_{f}$ is the old rotation rate of the frame, and $\Omega_{f}^{\prime}$ the new rate, and where the superscripts $b$ and $c$ denote respectively the value of the azimuthal velocity at the beginning of cells $5 b$ and $5 \mathrm{c}$ of Fig. 2. which are also the values at the beginning and end of the velocity correction routine (second part of cell $5 \mathrm{~b}$ of Fig. 2p. The geometrical terms of Eq. (44) are evaluated at the center of the faces where $v_{\phi}$ is defined. This substep trivially conserves angular momentum to machine accuracy.

The flux evaluation is done through an upwind method to infer the value of the quantity $Q^{*}$ at the center of the face at half time step. While there has been a trend in the past decade to turn to Riemann's problem to evaluate the fluxes at the interface in many astrophysical fluid dynamics codes, as initially devised by Godunov (1959), we make use here of the staggering of the velocity field to express simply the flux as:

$$
\mathcal{F}_{X_{i+1 / 2}^{n+1 / 2}}^{n}=v_{X i+1 / 2}\left[Q_{i+1 / 2}^{* x}\right]^{n+1 / 2} \mathcal{S}_{i+1 / 2}
$$

where the subscript $X$ represent the direction normal to the face, along which the flux is evaluated, and where $Q_{i+1 / 2}^{* x}$ is the $x$-interpolated value of the cell centered quantity $Q$ onto the face $i+1 / 2$, at the middle of the time step. In Eq. 45 we have omitted the $j, k$ subscripts for the sake of brevity, since the flux calculation only involves operations along one axis. As in Stone \& Norman (1992a), we consider a unique characteristic speed which is the velocity of the flow at the interface, at the beginning of the time step, given directly by $v_{x i+1 / 2 j k}^{n}$. This characteristic is integrated back in time over $\Delta t / 2$, and the interpolated value $Q^{*}$ of the variable $Q$ under consideration is sought at the location $x_{*}$ thus reached, which is:

$$
x_{*}=x_{i+1 / 2}-v_{x i+1 / 2}^{n} \Delta t / 2 .
$$

The interpolation, regardless of its order, naturally contains a test on the velocity sign, since $x_{*}$ falls in cell $i$ if $v_{x i+1 / 2}^{n}<0$, and in cell $i+1$ otherwise. Eq. (45) can finally be recast as:

$$
\mathcal{F}_{X_{i+1 / 2}^{n+1 / 2}}^{n}=v_{X_{i+1 / 2}^{n}}^{n}\left(Q^{* x}\right)_{i+1 / 2} \mathcal{S}_{i+1 / 2} .
$$

For the interpolated value of $Q$, we use a zone-wise linear reconstruction using van Leer's slopes (van Leer 1977) for most of our sub steps ${ }^{8}$. There is an exception to this: when we perform the uniform residual step of orbital advection (which we will present in section 3.5.1, we use a zone-wise parabolic reconstruction of the field, using the so-called PPA or Piecewise parabolic advection algorithm ${ }^{9}$, in a manner similar to what is done in the PLUTO code (Mignone et al. 2012). For the sake of completeness we give hereafter the detail of the evaluation of the interpolated value using van Leer's slopes:

$$
Q_{i+1 / 2}^{* x}=\left\{\begin{array}{cl}
Q_{i}+a_{i}\left(\Delta X_{i}-v_{x i+1 / 2} \Delta t\right) / 2 & \text { if } v_{x i+1 / 2} \geq 0 \\
Q_{i+1}-a_{i+1}\left(\Delta X_{i}+v_{x i+1 / 2} \Delta t\right) / 2 & \text { if } v_{x i+1 / 2}<0,
\end{array}\right.
$$

where $a_{i}$ is van Leer's slope, given by:

$$
a_{i}=\left\{\begin{array}{cl}
0 & \text { if } \Delta Q_{i+1 / 2} \Delta Q_{i-1 / 2}<0 \\
2 \frac{\Delta Q_{i+1 / 2} \Delta Q_{i-1 / 2}}{\Delta Q_{i+1 / 2}+\Delta Q_{i-1 / 2}} & \text { otherwise }
\end{array}\right.
$$

with $\Delta Q_{i+1 / 2}=\left(Q_{i+1}-Q_{i}\right) / \Delta X_{i}$. This evaluation of the interface value at half time step corresponds to cell $12 \mathrm{~b}$ of Fig. 2 During this substep, the interface values of density, energy and momenta are evaluated at the cell interfaces.

We use consistent transport (Norman et al. 1980, Stone \& Norman 1992a) for all quantities other than mass ${ }^{10}$.

\subsubsection{Momenta advection}

As discussed in section 3.2, we must transport a number of momenta-like quantities. These momenta do not have a trivial definition on a staggered mesh, as they involve the product of quantities which have different centering. In contrast with what is done in the ZEUS code (Stone \& Norman 1992a), where the control volumes of staggered quantities are shifted and involve zone centered fluxes along the staggered direction, we define two flavors of each momentum, that we call the left and right momenta, which we define respectively as:

$$
\begin{aligned}
& \Pi_{i}^{-}=\rho_{i} v_{i-1 / 2} \\
& \Pi_{i}^{+}=\rho_{i} v_{i+1 / 2},
\end{aligned}
$$

which we transport as any cell-centered quantity. Eq. (49) and (50) involve the product of the cell-centered density $\rho_{i}$ and respectively of the left (right) interface value of velocity $v_{i-1 / 2}\left(v_{i+1 / 2}\right)$. These momenta are evaluated when entering the set of transport sub steps, which corresponds to cell $12 \mathrm{a}$ of Fig. 2. Upon completion of the transport sub steps, the new velocity is inferred from the new momenta and new density as follows:

$$
v_{i-1 / 2}^{n+1}=\frac{\Pi_{i-1}^{+}{ }^{n+1}+\Pi_{i}^{-n+1}}{\rho_{i-1}^{n+1}+\rho_{i}^{n+1}},
$$

where we note that Eq. (51) applied to the old momenta and density yields the old velocity. This transformation corresponds to cell $12 \mathrm{~d}$ of Fig. 2. Eqs. (49) and (51) are used in 
the Cartesian case. In other geometries we amend them according to Tab. 2. Our procedure ensures the conservation to machine accuracy of both the left and right momentum, and therefore of the discrete momentum defined as:

$$
\Pi_{i}=\frac{1}{2}\left(v_{i-1 / 2}+v_{i+1 / 2}\right) \rho_{i},
$$

which appears as the arithmetic mean of left and right momenta. When used with consistent transport, the technique of left and right momenta advection is tantamount, for the transport of $\Pi_{i}$, to evaluating the interpolated velocity $v^{*}$ at the left interface as the arithmetic average of the zone centered interpolates $v_{i}^{*}$ and $v_{i+1}^{*}$. Its main difference with respect to the method used in the ZEUS code is therefore that in the latter the mass fluxes are averaged to yield zone centered mass fluxes, whereas in our method it is the star values that are averaged in order to keep the mesh zones as control volumes. This avoids, in particular, the averaging of mass fluxes, and makes the transport of momenta exactly consistent with the transport of zone centered quantities. Besides, it is important that the control volume be the same for all variables when using the orbital advection (aka FARGO) algorithm, which is the subject of section 3.5.1.

\subsubsection{Momentum conservation in our implementation}

The previous section shows that momenta are conserved to machine accuracy during the transport step. In codes based on Godunov's method, the pressure at the interface between zones is included in the momenta fluxes, which ensures that momenta are conserved to machine accuracy during a full update. In our case, however, the pressure gradient is dealt with apart from the transport step, in substep 1 (see section 3.3, and it is necessary to address separately the conservation of momenta under the action of this source term. Since it is a case of particular interest for FARGO3D, we specialize our discussion to the case of the angular momentum, in spherical coordinates, for a rotating frame. The generalization to other components of momentum is straightforward.

During the pressure source step, the following transformation is applied to the azimuthal velocity (note that all the discussion in this section applies at fixed values of $r_{j}$ and $\sin \theta_{k}$, so we drop for the rest of this section the $j$ and $k$ notation):

$$
\frac{v_{i-1 / 2}^{n+a}-v_{i-1 / 2}^{n}}{\Delta t} \equiv \frac{\Delta v_{i-1 / 2}}{\Delta t}=-2 \frac{P_{i}-P_{i-1}}{\Delta x\left(\rho_{i}+\rho_{i-1}\right)},
$$

where $P_{i}$ is the pressure defined in the center of cell $i, \Delta t$ is the time step and $\Delta x=r \Delta \phi$ the cell width. During the source step, the integrated zone centered momentum therefore varies of the quantity:

$$
\sum_{i=0}^{N-1} \Delta \Pi_{i} \Delta x=-r \sin \theta \Delta t\left(\sum_{i=0}^{N-1} \frac{P_{i}-P_{i-1}}{\rho_{i}+\rho_{i-1}} \rho_{i}+\frac{P_{i+1}-P_{i}}{\rho_{i+1}+\rho_{i}} \rho_{i}\right),
$$

where

$$
\Delta \Pi_{i}=\frac{1}{2}\left(\Delta \Pi_{i}^{-}+\Delta \Pi_{i}^{+}\right)
$$

is the variation of the zone centered momentum in zone $i$ during the source step. We have:

$$
\begin{aligned}
\Delta \Pi_{i}^{ \pm} & =\Delta\left[\rho_{i}\left(r v_{\phi}^{i \pm 1 / 2} \sin \theta+r^{2} \sin ^{2} \theta \Omega_{f}\right)\right] \\
& =\rho_{i} r \sin \theta \Delta v^{i \pm 1 / 2} .
\end{aligned}
$$

Renumbering the second sum of the right hand side of Eq. 54) from 1 to $N$, we are exclusively left with edge terms:

$\sum_{i=0}^{N-1} \Delta \Pi_{i} \Delta x=r \sin \theta \Delta t\left(\frac{P_{0} \rho_{-1}+P_{-1} \rho_{0}}{\rho_{0}+\rho_{-1}}-\frac{P_{N-1} \rho_{N}+P_{N} \rho_{N-1}}{\rho_{N-1}+\rho_{N}}\right)$,

which cancel each other if we account for the mesh periodicity in azimuth: $\rho_{-1}=\rho_{N-1}, \rho_{N}=\rho_{0}$, and similar relations for the pressure:

$$
\sum_{i=0}^{N-1} \Delta \Pi_{i} \Delta x=0
$$

The angular momentum is therefore conserved to machine accuracy, regardless of whether the frame is rotating. A corollary of this is that shock jump conditions are satisfied in isothermal setups, even if the pressure contains an artificial viscosity, and the production of vortensity that occurs when a fluid parcel crosses a shock, which is governed by the shock jump conditions, is also captured correctly by the code, as was noted by Lin \& Papaloizou (2010).

The conservation of momentum under the pressure source term is due to the fact that the value of the density evaluated at the zone interface that we use in Eq. (53) is the arithmetic mean of the adjacent zone centered values. This conservation property can be generalized to any source term arising from the gradient of a zone centered quantity divided by the density.

\subsection{Stability}

The integration time step $\Delta t$ over which a full cycle of (magneto)hydrodynamical sub-steps is performed has to be limited in order to ensure stability of the explicit methods we use. This condition is known as the Courant-Friedrichs-Levy (CFL) condition, or the Courant condition. Its broad physical meaning is that information cannot travel over more than one cell per time step. Although in simple cases it is possible to determine exactly the stability criterion with a von Neumann and Richtmyer's analysis, in general, as is the case here, we resort to heuristic methods to work out the maximum allowed time step. Following Stone \& Norman (1992a), we take as the maximum time step allowed ${ }^{11}$ :

$$
\Delta t=C \min \left\{\left(\sum_{i} \Delta t_{i}^{-2}\right)^{-1 / 2}\right\}
$$

where $C$ is a real parameter smaller than one, called the Courant number. Unless specified otherwise we use $C=0.44$, a value that we regard as a good compromise between speed and stability for our test problems. In Eq. (59), the minimum is sought over the full computational domain (excluding ghost zones), which corresponds to cell 6 of the flow chart of Fig. 2. The different $\Delta t_{i}$ correspond to different processes that individually limit the time step. We draw hereafter the list of these individual time steps. The $j$ index below represents each direction of the mesh $(x y z / \phi r z / \phi r \theta)$ or a subset of those in lower dimension. In a multidimensional case we set $\Delta t_{i}$ as $\min _{j}\left\{\Delta t_{i, j}\right\}$.

1. Sound or magnetosonic waves: $\Delta t_{1}=\Delta_{j} / C_{w}$, where $C_{w}$ is the maximum speed of the waves that can propagate in the medium. In the MHD case, it is the fast magnetosonic wave, with expression $C_{w}^{2}=C_{s}^{2}+v_{A}^{2}$, where $C_{s}$ is the speed of sound and $v_{A}$ is the Alfvén velocity: $v_{A}^{2}=B^{2} /\left(\mu_{0} \rho\right)$. In the purely $\mathrm{HD}$ case $C_{w}$ is simply $C_{s}$. 
2. Fluid motion: $\Delta t_{2}=\Delta_{j} /\left|V_{j}\right|$.

3. Artificial viscosity: $\Delta t_{3}=C_{2}\left|\Delta_{j} / \Delta v_{j}\right|$, where we choose the constant value $C_{2}=4 \sqrt{2}$. $\left(\Delta v_{j}\right)$ is the difference between successive values of $v_{j}$ along the $j$ direction (e.g. $\Delta v_{x}=v_{x i+1 / 2}-v_{x i-1 / 2}$ ).

4. Viscosity: $\Delta t_{4}=\Delta_{j}^{2} /(4 v), v$ being the kinematic viscosity.

5. Resistivity: $\Delta t_{5}=\Delta_{j}^{2} /(4 \eta), \eta$ being the resistivity.

\subsubsection{Orbital advection}

The transport step presented in section 3.4 is known to yield the following issues:

1. Large nearly uniform azimuthal velocities severely limit the time step (see section 3.5)

2. Truncation errors depend on the frame of reference (Robertson et al.|2010).

We present here a derivation which is similar to that of Masset (2000), except for the use of Piecewise Parabolic Advection to perform the fractional shift. The main idea of orbital advection techniques is to decompose, for each ring of cells at a given radius and colatitude, the azimuthal velocity into a large, uniform velocity and a residual, smaller velocity (which is tantamount to working in a nearly corotating frame in each ring):

$$
v=v^{0}+\delta v,
$$

where $v \equiv v_{\phi}$ in spherical and cylindrical geometries, and $v_{x}$ in the Cartesian (shearing sheet) case. The transport equation in the azimuthal direction, which reads

$$
\partial_{t} Q+\partial_{x}(v Q)=0
$$

where $\partial_{x} \equiv(1 / r) \partial_{\phi}$ in cylindrical geometry and $\partial_{x} \equiv$ $(1 / r \sin \theta) \partial_{\phi}$ in spherical geometry. Eq. (61) is solved in two steps, using the operator splitting technique:

$$
\begin{array}{r}
\partial_{t} Q+\partial_{x}(\delta v Q)=0 \\
\partial_{t} Q+v^{0} \partial_{x} Q=0
\end{array}
$$

Eq. (62) is solved using the upwind method described in section 3.4. Eq. 63) amounts to a shift of the initial profile:

$$
Q(x, t+\Delta t)=Q\left(x-v^{0} \Delta t, t\right) .
$$

Since this shift does not necessarily represent an integer number of zones, we further split Eq. (63) into two sub steps. We decompose $v^{0}$ as:

$$
v^{0}=v_{\text {shift }}+v_{\text {shift }}^{R},
$$

where

$$
v_{\text {shift }}=N \frac{\Delta X}{\Delta t}
$$

with $N=E\left(v^{0} \frac{\Delta t}{\Delta X}\right), E(X)$ being the nearest integer to $X$. Using again the operator splitting technique, Eq. 63) is equivalent to solving in succession ${ }^{12}$ :

$$
\partial_{t} Q+v_{\text {shift }} \partial_{x} Q=0
$$

and

$$
\partial_{t} Q+v_{\text {shift }}^{R} \partial_{x} Q=0 .
$$

The solution of Eq. 67) is given by

$$
\begin{aligned}
Q_{i}^{n+1} & \equiv Q\left(x_{i}, t+\Delta t\right)=Q\left(x-v_{\text {shift }} \Delta t, t\right) \\
& =Q\left(x_{i}-N \Delta X, t\right)=Q_{i-N}^{n},
\end{aligned}
$$

which is simply a circular permutation of the zone values within the ring under consideration.

When using orbital advection, the velocity to be used in Eq. (48) is the residual velocity $\delta v$. Similarly, Eq. (68) can be recast as:

$$
\partial_{t} Q+\partial_{x}\left(v_{\text {shift }}^{R} Q\right)=0
$$

and can therefore be dealt with using the same advection machinery. We perform this fractional uniform advection using a higher order interpolation (Piecewise Parabolic Advection). The interface values are given by:

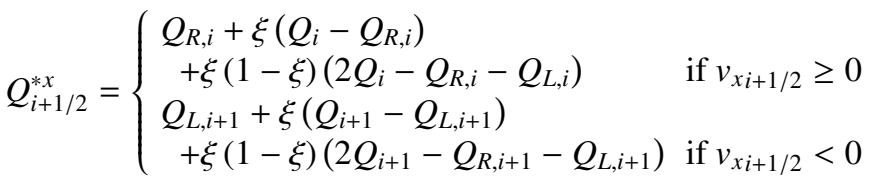

where $\xi=v_{i+1 / 2} \Delta t / \Delta x$, and $Q_{L / R}$ are the monotonized left/right interface values for $Q$, obtained as follows.

From $\delta Q_{i}=\left(Q_{i+1}-Q_{i-1}\right) / 2$, we firstly define a monotonized centered slope in each cell with:

$$
\delta_{m} Q_{i}=\left\{\begin{array}{l}
0 \quad \text { if }\left(Q_{i+1}-Q_{i}\right)\left(Q_{i}-Q_{i-1}\right) \leq 0 \\
\min \left(2\left|Q_{i}-Q_{i-1}\right|, 2\left|Q_{i+1}-Q_{i}\right|,\left|\delta Q_{i}\right|\right) \\
\quad \times \operatorname{sgn}\left(\delta Q_{i}\right) \quad \text { otherwise, }
\end{array}\right.
$$

This procedure corresponds to that of Colella \& Woodward (1984) with a uniform cell size. In a second stage, parabolic interpolations are used to reconstruct the quantity at the interfaces with:

$$
Q_{i+1}^{L}=Q_{i}^{R}=Q_{i}+\frac{1}{2}\left(Q_{i+1}-Q_{i}\right)-\frac{1}{6}\left(\delta_{m} Q_{i+1}-\delta_{m} Q_{i}\right),
$$

and final tests are warranted to remove local extrema potentially created by the above procedure, as follows:

$$
\begin{cases}Q_{i}^{L}=Q_{i}^{R}=Q_{i} & \text { if }\left(Q_{i+1}-Q_{i}\right)\left(Q_{i}-Q_{i-1}\right) \leq 0 \\ Q_{i}^{L}=3 Q_{i}-2 Q_{i}^{R} & \text { if } \Delta_{i} C_{i}>\Delta_{i}^{2} / 6 \\ Q_{i}^{R}=3 Q_{i}-2 Q_{i}^{L} & \text { if }-\Delta_{i}^{2} / 6>\Delta_{i} C_{i},\end{cases}
$$

where $\Delta_{i}=Q_{i}^{R}-Q_{i}^{L}$ and $C_{i}=Q_{i}-\left(Q_{i}^{L}+Q_{i}^{R}\right) / 2$. The use of this method requires one additional layer of ghost or buffer zones (extra zones outside the mesh that are used either to synchronize the data between different computing processes, or to prescribe boundary conditions) with respect to the use of van Leer's slope presented in section 3.4. We employ it exclusively to perform the fractional uniform residual of the orbital advection, as in Mignone et al. (2012). Along the orbital direction, no ghost zones are used, as the algebra on the corresponding zone index $(i)$ is hard coded to account for the mesh periodicity ${ }^{13}$. We also mention that we have tried to use the steepened version of the PPA method, as described by Colella \& Woodward (1984) at Eqs. (1.15) to (1.17), using the same numerical thresholds. This method is definitely not suitable for Keplerian disks, where it is found to spuriously generate small scale vortices.

The circular permutation of Eq. (69) does not introduce numerical errors, and $v_{\text {shift }}$ does not have to be included in the stability analysis: it does not contribute to the Courant condition. By construction of $N$, we have $\left|v_{\text {shift }}^{R} \Delta t\right|<1 / 2$, so that $v_{\text {shift }}^{R}$ does not enter the Courant condition either. 
The Courant condition is thus determined by a criterion similar to that of Eq. (59), in which we replace the azimuthal velocity $v$ by $\delta v$. So far we have not specified how the decomposition of Eq. (60) is performed. It should be done so that the residual velocity $\delta v$ has the smallest possible $\mathcal{L}_{\infty}$ norm, which is realized if one adopts:

$$
v_{j k}^{0}=\frac{1}{2}\left[\max \left(v_{i j k}\right)+\min \left(v_{i j k}\right)\right],
$$

where the minimum and maximum values are sought with $j$ and $k$ being fixed, for all values of $i$. Most implementations of the FARGO algorithm consider instead the azimuthal average (Masset 2000; Kley et al. 2009; Mignone et al. 2012). We suggest that some difference might be expected when the perturbed velocity is significantly larger than the sound speed (so it is what primarily limits the time step), which happens, in the context of planets in disks, when giant planets are present. Tests we have performed in two-dimensional disks with an aspect ratio of $5 \%$ and a Jupiter mass planet have shown differences at the percent level between the execution times of the two implementations, hence the azimuthal average is good enough for most applications.

We conclude this section with the remark that we apply the orbital advection algorithm to the same variables presented in section 3.2. The orbital advection, which consists of a circular permutation of indices and conservative updates involving azimuthal fluxes similar to those of section 3.4 conserves therefore these fields to machine accuracy. Just as its predecessor code (FARGO), the FARGO3D code conserves mass and angular momentum to computer accuracy.

As pointed out in section 3.4.1 the technique of left and right momenta allows us to have the same control volume for all the variables transported. This is an important prerequisite to implement orbital advection in staggered mesh codes. With the standard method of the ZEUS code, for instance, we would have to consider rings staggered in radius for the transport of radial momentum, so that the implementation of orbital advection would be far more involved. To the best of our knowledge, all implementations of the FARGO algorithm in staggered mesh codes make use of the left and right momenta technique. Codes based on higher order Godunov methods do not suffer from this problem, since all their hydrodynamics variables are zone centered (eg Mignone et al.2012).

Orbital advection must also be implemented to solve the induction equation in the MHD case. This is presented in section 3.7 .

\subsubsection{Amendments to the CFL condition}

The large, axisymmetric azimuthal velocity $v_{0}$ of Eq. (60) does not enter into play in the stability criterion of Eq. (59). Instead, one must substitute the total azimuthal velocity by the residual velocity $\delta v$ in the CFL criterion (in the second item of the list of section 3.5. Since the limit timestep must be evaluated before entering the different (magneto-)hydrodynamical substeps, and since the evaluation of the limit timestep requires the knowledge of the bulk azimuthal velocity for orbital advection, the determination of this quantity is one of the first tasks performed during a full update. It corresponds to cell 3 of the flow chart of Fig. 2. In geometrically thin disks, which have an aspect ratio $h=c_{s} / v_{K} \ll 1$, the large orbital velocity usually dominates largely the Courant condition. By getting rid of orbital motion in the latter, we can leverage the time step by a factor $\sim h^{-1}$ for weakly perturbed flows. This ratio typically amounts to an order of magnitude in protoplanetary disks. In addition to relaxing this constraint on the time step, we introduce a new time step limit linked to the shear, intended to prevent radially neighboring zones from becoming disconnected after one time step. This criterion reads:

$$
\Delta t_{\text {shear }}<C_{0}\left(\frac{V_{X}(i, j, k)}{\Delta X_{j}}-\frac{V_{X}(i, j+1, k)}{\Delta X_{j+1}}\right)^{-1},
$$

where we impose that the content of radially neighboring zones are offset at most a fraction $1-C_{0}$ of the azimuthal zone width after advection during one time step along the orbital motion. In practice, this criterion is seldom useful. The shear could become an issue in the inner regions of the mesh, where the cells can be very elongated radially $(\Delta r>r \Delta \phi)$. Omitting for simplicity the multiplication by the Courant number, the shear time limit will be important only if it is smaller than other time limits (see section 3.5), in particular the time limit imposed by sound waves, which reads here $\Delta t_{\text {sound }}=r \Delta \phi / C_{s}$. Specializing to Keplerian shear, we have here $\Delta t_{\text {shear }}=r \Delta \phi /(3 / 2 \Omega \Delta r)$. The shear limit will therefore supersede the usual time step limit if $\Delta r>2 \mathrm{H} / 3$, that is to say if the pressure scale length is unresolved, a situation which should be avoided in any case. We note that if the cells keep an aspect ratio close to unity near the inner boundary, the shear time step limit is of the order of the local orbital time, way larger than other limits.

\subsection{Magnetohydrodynamics}

To evolve numerically the magnetic field, we follow the Method of Characteristics + Constrained transport (MOCCT) presented in detail by Hawley \& Stone (1995). The CT method, developed by Evans \& Hawley (1988), ensures a constant (here, null) divergence of the magnetic field to machine accuracy. This method requires the values at half time step of the velocity and magnetic field components at the middle of a zone edge. The MOC is used to calculate these values. Instead of considering a unique characteristic velocity as is done for the HD quantities (see section 3.4), a more sophisticated approach is adopted in which incompressible transverse Alfvén waves are considered. Leftward and rightward propagating Alfvén waves carry different eigenvalues which are evaluated at the foot of their respective characteristics, at the beginning of the time step, and combined at half time step to yield the velocity and magnetic field values at the zone edge (Stone \& Norman 1992b). The discussion in the following sections cover the substeps of cells 8,10 and 11 of Fig. 2

\subsubsection{Constrained transport method}

The main idea of the constrained transport (CT) method, formulated by Evans \& Hawley (1988), is to ensure that the divergence of the magnetic field is maintained constant (here, naturally zero) for all time, at machine accuracy, using a conservative formulation for its flux based on the evaluation of electromotive forces. Insomuch as the sharing of fluxes on the zone faces satisfies a discrete version of the divergence theorem, sharing electromotive forces (electric fields) at the zone edges satisfies a discrete version of Stoke's theorem. The integral form of Eq. (5) reads:

$$
\frac{\partial \mathcal{F}^{B}}{\partial t}=\int_{\partial S}(\vec{v} \times \vec{B}-\eta \nabla \times \vec{B}) \cdot d \vec{l}
$$

where $\mathcal{F}^{B}$ is the magnetic flux through a surface $S$ (bounded by $\partial S$ ). An approximation for the flux for the $X$-component 
of the magnetic field across a zone face is:

$$
\mathcal{F}_{X i+1 / 2}^{B}=B_{X i+1 / 2} \mathcal{S}_{i+1 / 2},
$$

with straightforward generalization to the $Y$ and $Z$ components. We recall that the magnetic field is face centered like the velocity field, as depicted in Fig. 1. The method to satisfy the flux conservation is to derive the rate of change of the magnetic fluxes by evaluating the circulation of the electromotive force (EMF) defined by $\vec{\varepsilon}=\vec{v} \times \vec{B}$. The $X(Y, Z)$ component of the EMF is defined at the middle of the edge along the $X(Y, Z)$ direction. The flux evolution is given by:

$$
\begin{aligned}
\frac{\mathcal{F}_{X i+1 / 2, j, k}^{B^{n+1}}-\mathcal{F}_{X i+1 / 2, j, k}^{B^{n}}}{\Delta t} & =\varepsilon_{i+1 / 2, j, k+1 / 2}^{Y} \Delta Y_{i+1 / 2, j, k+1 / 2} \\
& -\varepsilon_{i+1 / 2, j, k-1 / 2}^{Y} \Delta Y_{i+1 / 2, j, k-1 / 2} \\
& -\varepsilon_{i+1 / 2, j+1 / 2, k}^{Z} \Delta Z_{i+1 / 2, j+1 / 2, k} \\
& +\varepsilon_{i+1 / 2, j-1 / 2, k}^{Z} \Delta Z_{i+1 / 2, j-1 / 2, k},
\end{aligned}
$$

and similar relations for the $Y$ and $Z$ components of the flux are obtained with a circular permutation $X \rightarrow Y \rightarrow Z \rightarrow X$ and $i \rightarrow j \rightarrow k \rightarrow i$. By summing the variations of $\mathcal{F}_{X i+1 / 2, j k}^{B}$, $\mathcal{F}_{Y i, j \pm 1 / 2, k}^{B}$ and $\mathcal{F}_{Z i, j, k \pm 1 / 2}^{B}$ (six terms in total), we can see that each edge is swept twice in opposite directions, yielding a null net flux variation of the magnetic field across the zone boundary. If it is initially divergence free, its divergence vanishes at all times.

\subsubsection{Method of characteristics}

The integrand of Eq. (74) features two terms. The first one corresponds to the field induction in ideal MHD, and the second one to resistive effects. We focus hereafter on the first term. The resistive term is added afterwards, prior to the constrained transport substep. As seen in section 3.6.1, the components of the electromotive force are defined along the edges. In order for the constraint transport step to be second order accurate in time, they must be specified at time $t+\Delta t / 2$. We consider specifically hereafter the case of the $Z$-component of the electromotive force. Other components can trivially be deduced by the circular permutations given in section 3.6.1 We have:

$$
\varepsilon_{Z}=v_{X} B_{Y}-v_{Y} B_{X},
$$

and $\varepsilon^{Z}$ is defined at $(i+1 / 2, j+1 / 2, k)$, hence we need the interpolated values $v_{X, Y_{i+1 / 2, j+1 / 2, k}^{*}}$ and $B_{X, Y}^{*} Y_{i+1 / 2, j+1 / 2, k}^{*}$ at time $t+\Delta t / 2$. These are obtained using a method of characteristics which considers a restricted problem for each pair $\left(v_{X}^{*}, B_{X}^{*}\right)$ and $\left(v_{Y}^{*}, B_{Y}^{*}\right)$. The $X$-components are calculated by considering a $1.5 \mathrm{D}$ problem along the direction $Y$, and vice-versa ${ }^{14}$. We consider below the case of the $Y$-components:

$$
\begin{aligned}
\frac{\partial v_{Y}}{\partial t}+v_{X} \frac{\partial v_{Y}}{\partial X} & =\frac{B_{X}}{\mu_{0} \rho} \frac{\partial B_{Y}}{\partial X}, \\
\frac{\partial B_{Y}}{\partial t}+v_{X} \frac{\partial B_{Y}}{\partial X} & =B_{X} \frac{\partial v_{Y}}{\partial X} .
\end{aligned}
$$

Eq. (78) corresponds to the $Y$ projection of Eq. (2) in which we neglect the derivatives along the $Y$ and $Z$ coordinates, and all source terms other than the magnetic tension. Eq. (79) corresponds similarly to the $Y$ projection of Eq. (5) in which we neglect resistive effects, and derivatives along the $Y$ and $Z$ coordinates. This procedure only retains the non-compressive,

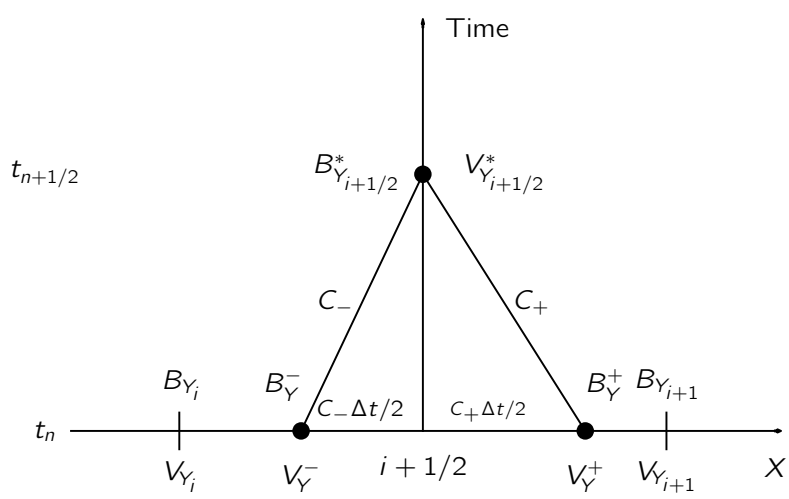

FIG. 3.- Space-time diagram showing Alfvén's characteristics used to the extrapolate the EMF components, for the case $B_{X}>0$.

Alvén waves that may exist if $B_{X}$ does not vanish. By adding $\pm\left(\mu_{0} \rho\right)^{-1 / 2}$ times Eq. (79) to Eq. (78), we find the characteristic equations:

$$
\partial_{t} \psi_{ \pm}+C_{ \pm} \partial_{X} \psi_{ \pm}=0
$$

where the characteristic speed is

$$
C_{ \pm}=v_{X} \mp \frac{B_{X}}{\sqrt{\mu_{0} \rho}},
$$

and where the eigenvalue $\psi_{ \pm}$is:

$$
\psi_{ \pm}=v_{Y} \pm \frac{B_{Y}}{\sqrt{\mu_{0} \rho}} .
$$

The eigenvalues $\psi_{+}$and $\psi_{-}$are therefore conserved along the characteristics with respective speeds $C_{+}$and $C_{-}$. Their value is sought at the foot of these characteristics, using a piecewise linear reconstruction of $v_{Y}$ and $B_{Y}$ with van Leer's slope, exactly as done in the transport step (section 3.4 for the characteristic with the flow speed. The method is depicted in Fig. 3. In our implementation, the values of $v_{X}$ and $B_{X}$ used to evaluate the characteristic speeds in Eq. (81) are obtained themselves with an upwind interpolation of these fields in $Y$ (again making use of a zone-wise reconstruction with van Leer's slopes) with the flow speed $\overline{v_{Y}}$, defined as the arithmetic average of $v_{Y i j-1 / 2 k}$ and $v_{Y i-1 j-1 / 2 k}$. We note these values $v_{x}^{u}$ and $B_{x}^{u}$, where the $u$ superscript, which conveys the upwind method, is used instead of $*$ to avoid confusion with the values obtained through the MOC. The value of $\rho$ used in Eqs. (81) and (82) is the arithmetic average of the four values $\rho_{i j k}, \rho_{i-1 j k}, \rho_{i j-1 k}$ and $\rho_{i-1 j-1 k}$. Finally, the star value $v_{Y}^{*}$ and $B_{Y}^{*}$ are given by:

$$
\begin{aligned}
& v_{Y}^{*}=\frac{1}{2}\left(\psi_{+}+\psi_{-}\right)=\frac{1}{2}\left(v_{Y}^{+}+v_{Y}^{-}+\frac{B_{Y}^{+}-B_{Y}^{-}}{\sqrt{\mu_{0} \rho}}\right) \\
& B_{Y}^{*}=\frac{\sqrt{\mu_{0} \rho}}{2}\left(\psi_{+}-\psi_{-}\right)=\frac{1}{2}\left[B_{Y}^{+}+B_{Y}^{-}+\sqrt{\mu_{0} \rho}\left(v_{Y}^{+}-v_{Y}^{-}\right)\right] .
\end{aligned}
$$

where $v^{ \pm}$and $B^{ \pm}$are the field values at time $t$ at the foot of the corresponding characteristics. This corresponds to cell 8a of Fig. 2 The values given by Eq. (83) are used to evaluate the electromotive force by use of Eq. (777). Nonetheless, in order to avoid anomalous EMFs near rotational discontinuities of the magnetic field, we use the star values only with the terms 
they are consistent with, which are the upwind estimates of the transverse fields used to infer the characteristics speeds (Hawley \& Stone 1995). Eq. (77) then takes the form:

$$
\varepsilon_{Z}=\frac{1}{2}\left(v_{X}^{*} B_{Y}^{u}+B_{Y}^{*} v_{X}^{u}-v_{Y}^{*} B_{X}^{u}-B_{X}^{*} v_{Y}^{u}\right) .
$$

This corresponds to cell $8 \mathrm{~b}$ of Fig. 2 Prior to applying the constrained transport through Eq. (76) (and those obtained through circular permutations), which corresponds to cell 10 of Fig. 2. we add the resistive term to each component, that is to say the second term of the right hand side of Eq. (74). No special care has to be observed to evaluate the curl of the magnetic field at this stage: we use a simple finite difference estimate. This is done in substep 8c of Fig. 2 .

Lastly, prior to the update of the field, the source term corresponding to the Lorentz force is applied to each component of the velocity. Consider the update of the $X$ component. In Cartesian coordinates, the partial differential equation corresponding to this substep is:

$$
\partial_{t} v_{X}=\frac{1}{\mu_{0} \rho}\left(B_{Y} \partial_{Y} B_{X}+B_{Z} \partial_{Z} B_{X}-B_{Y} \partial_{X} B_{Y}-B_{Z} \partial_{X} B_{Z}\right)
$$

We note in this equation that the term $B_{X} \partial_{X} B_{X}$ arising from $\vec{B} . \nabla B_{X}$ cancels out with the corresponding term of the magnetic pressure gradient $-\partial_{X} B^{2} / 2$. We deal with the first two terms of the right hand side of Eq. (85) in a specific substep, which we detail below. The remaining two terms are dealt with in the substep 1 described in section 3.3 , the gradient of the zone centered two-point average of the transverse terms is applied in much the same way as the thermal pressure gradient. This is done at the beginning of the time step (in cell 7a of Fig. 2, with the value of the magnetic field at time $t$.

The magnetic tension source term, corresponding to the first two terms of Eq. (85), use the star value of the $B_{X}$ field on the edge of the face at $i-1 / 2$ to evaluate the gradients. The corresponding substep reads therefore:

$$
\begin{aligned}
& \frac{v_{X i-1 / 2 j k}^{c}-v_{X i-1 / 2 j k}^{b}}{\Delta t}=\frac{1}{\mu_{0}} \frac{2}{\rho_{i j k}+\rho_{i-1 j k}} \times \\
& \left(\overline{B_{Y}} \frac{B_{X i-1 / 2 j+1 / 2 k}^{*}-B_{X i-1 / 2 j-1 / 2 k}^{*}}{\Delta Y_{i-1 / 2 k}}+\right. \\
& \left.\overline{B_{Z}} \frac{B_{X i-1 / 2 j k+1 / 2}^{*}-B_{X i-1 / 2 j k-1 / 2}^{*}}{\Delta Z_{i-1 / 2 j}}\right) \text {. }
\end{aligned}
$$

The momentum transport is dealt with during the transport step of the full update (in cells 12 a to $12 \mathrm{~d}$ of Fig. 2), and similarly, the transport of the magnetic field is dealt with during the constrained transport update (cell 10 of Fig. 2). There is therefore no need to use an upwind method to seek the values of the magnetic field to be used in Eq. (86). The fluid element sitting at a face edge at the beginning of the time step, and the associated magnetic field, can be regarded as drifting together during the substep in which magnetic tension is applied. This has sometime been described as a Lagrangian substep (Hawley \& Stone 1995). As a consequence, the starred values in Eq. (86) are obtained with a method of characteristics in which the characteristic speed is only $\pm B_{X} / \sqrt{\mu_{0} \rho}$, instead of the speed given in Eq. (81). Similarly, we use for the bar values in Eq. (86) the suitable four-point averages rather than advection upwind estimates as in Eq. 84,. The different notation (superscript $u$ versus bar) is meant to convey this difference. The evaluation of the Lorentz force, and the corresponding update of velocities, are performed in cell $8 \mathrm{~d}$ of Fig. 2

Finally, when the mesh is non-Cartesian, geometric source terms arise from the $\vec{B} \cdot \nabla \vec{B}$ term. Those are also included in substep 1, i.e. in cell 7a of Fig. 2 For the sake of completeness we hereafter indicate the partial differential equations corresponding to this update.

\section{Cylindrical coordinates:}

$$
\begin{gathered}
\partial_{t} v_{\phi}=\frac{B_{r} B_{\phi}}{\mu_{0} \rho r} \\
\partial_{t} v_{r}=-\frac{B_{\phi}^{2}}{\mu_{0} \rho r}
\end{gathered}
$$

\section{Spherical coordinates:}

$$
\begin{aligned}
\partial_{t} v_{\phi} & =\frac{B_{r} B_{\phi}+B_{\theta} B_{\phi} \cot \theta}{\mu_{0} \rho r} \\
\partial_{t} v_{r} & =-\frac{B_{\phi}^{2}+B_{\theta}^{2}}{\mu_{0} \rho r} \\
\partial_{t} v_{\theta} & =\frac{B_{r} B_{\theta}}{\mu_{0} \rho r}-\frac{B_{\phi}^{2} \cot \theta}{\mu_{0} \rho r}
\end{aligned}
$$

\subsection{FARGO algorithm in MHD - Orbital Advection}

The generalization of the orbital advection (aka FARGO algorithm) to the MHD equations has first been considered by Johnson et al. (2008). Subsequently, a method that generalizes the CT method and therefore automatically ensures the preservation of the divergence free property of the magnetic field was devised by Stone \& Gardiner (2010). To the best of our knowledge, our implementation is the first implementation of this method in a staggered mesh code. Other implementations of the orbital advection of the magnetic field were with centered methods (Stone \& Gardiner 2010, Mignone et al. 2012). Our implementation does not require any adaptation, though: the centering of the magnetic field is the same in both kinds of codes, and they only differ by how the orbital advection of hydrodynamical quantities is implemented.

The following discussion sums up the principles of the method of Stone \& Gardiner (2010), and shows that it amounts to an azimuthal shift of the components of the magnetic field, similar to those of the hydrodynamics variables (see section 3.5.1), when there is no shear.

As for the hydrodynamics, orbital advection consists in splitting the set of governing equations into a set based on the residual velocity $\delta v$, and a set based on the orbital velocity, which is exclusively azimuthal, and axisymmetric. The first set is solved as detailed in the previous section. Orbital advection has been discussed in section 3.5.1. Its magnetic supplement corresponds to solving the following equation:

$$
\partial_{t} \vec{B}-\nabla \times\left(\overrightarrow{v_{0}} \times \vec{B}\right)=\overrightarrow{0},
$$

Component wise, Eq. (92) reads (the discussion hereafter specializes to the two-dimensional shearing sheet, but generalization to three dimensions in cylindrical or spherical coordinates is immediate):

$$
\begin{aligned}
& \partial_{t} B_{X}+v_{0} \partial_{X} B_{X}=q B_{Y} \\
& \partial_{t} B_{Y}+v_{0} \partial_{X} B_{Y}=0,
\end{aligned}
$$


where $q=\partial_{Y} v_{0}$ may depend on $Y$ but neither on $X$ nor on time, and where the constraint $\nabla \cdot \vec{B}=0$ has been used in the first of these equations, which state that the transverse field simply obeys an advection equation at the uniform speed $v_{0}$, and the shear of the transverse field acts as a source term on the longitudinal component, in addition to its advection. The system above trivially admits the following exact solution:

$$
\begin{aligned}
& B_{X}(x, t)=B_{X}^{0}\left(x-v_{0} t\right)+t q B_{Y}^{0}\left(x-v_{0} t\right) \\
& B_{Y}(x, t)=B_{Y}^{0}\left(x-v_{0} t\right),
\end{aligned}
$$

where $B_{X / Y}^{0}(x)$ are the $X$ and $Y$ components of the magnetic field at $t=0$. For any finite time interval $\Delta t$, we define the upstream average of the vertical electric field as:

$$
\overline{E_{z}}(x, t, \Delta t)=\frac{1}{\Delta x} \int_{x-\Delta x}^{x} v_{0} B_{y}\left(x^{\prime}, t\right) d x^{\prime},
$$

where $\Delta x=v_{0} \Delta t$. Using Eqs. (95) and 96, we have:

$$
-\partial_{x} \overline{E_{z}}(x, t, \Delta t)=\frac{1}{\Delta t}\left[B_{y}(x, t+\Delta t)-B_{y}(x, t)\right] .
$$

Similarly, we have:

$\partial_{y} \overline{E_{z}}(x, t, \Delta t)=q B_{y}(x-\Delta x, t)-\frac{1}{\Delta t}\left[B_{x}(x, t)-B_{x}(x-\Delta x, t)\right]$,

where we have used the property $\nabla \cdot \vec{B}=0$. Using Eq. (94), this result can be recast as:

$$
\partial_{y} \overline{E_{z}}(x, t, \Delta t)=\frac{1}{\Delta t}\left[B_{x}(x, t+\Delta t)-B_{x}(x, t)\right] .
$$

Eqs. (97) and (99) state that the upstream average of the electric field can be used to get the exact variation of the magnetic field, regardless of the magnitude $\Delta t$ of the time step. This is because the flow velocity is uniform along the streamlines and constant in time, so that the upstream average coincides with the Eulerian time average over the time step. With discretization in space, we perform the integral of Eq. 96, over as many zones as necessary to cover the arcs of length $\Delta x$. Only the $Y$ and $Z$ components of the electric field do not vanish. They are respectively staggered in $Z$ and $Y$. The velocity $v_{0}$ used in their evaluation is the two-point arithmetic average of the azimuthal velocities in the zones adjacent to the face considered. It may be enlightening to consider the special case in which there is no shear, and the arcs span an integer amount $N$ of cells in azimuth. Under these special circumstances, we have:

$$
\bar{E}_{z i-1 / 2 j-1 / 2}=\frac{v_{j-1 / 2}^{0}}{N} \sum_{m=1}^{N} B_{Y i-m j-1 / 2} .
$$

The update of the $Y$ component reads (all indices for the $Y$ direction have implicitly value $j-1 / 2$ ):

$$
\begin{aligned}
\Delta X \frac{B_{Y i}^{n+1}-B_{Y i}^{n}}{\Delta t} & =-\bar{E}_{z i+1 / 2}+\bar{E}_{z i-1 / 2} \\
& =\frac{v_{j-1 / 2}^{0}}{N}\left(-B_{Y i}^{n}+B_{Y i-N}^{n}\right) .
\end{aligned}
$$

Since by assumption $v_{j-1 / 2}^{0} \Delta t=N \Delta X$, we are simply left with:

$$
B_{Y i}^{n+1}=B_{Y i-N}^{n} .
$$

Similarly, the update of the $X$ component reads:

$$
\begin{aligned}
\Delta Y \frac{B_{X i-1 / 2}^{n+1}-B_{X i-1 / 2}^{n}}{\Delta t}= & \bar{E}_{z i-1 / 2 j+1 / 2}-\bar{E}_{z i-1 / 2 j-1 / 2} \\
= & \frac{v_{j+1 / 2}^{0}}{N} \sum_{m=1}^{N} B_{Y i-m j+1 / 2} \\
& -\frac{v_{j-1 / 2}^{0}}{N} \sum_{m=1}^{N} B_{Y i-m j-1 / 2}
\end{aligned}
$$

Using the no shear assumption, we can write $v_{j+1 / 2}^{0} / \Delta X_{j+1 / 2}=$ $v_{j-1 / 2}^{0} / \Delta X_{j-1 / 2}$, where $\Delta X_{j \pm 1 / 2}$ is the length of the upper (lower) edge in $X$ of the cell $(i, j)$. We therefore have:

$$
\begin{gathered}
\Delta Y \frac{B_{X i-1 / 2}^{n+1}-B_{X i-1 / 2}^{n}}{\Delta t}=(105) \\
\frac{v_{j+1 / 2}^{0}}{\Delta X_{j+1 / 2} N} \sum_{m=1}^{N} \Delta X_{j+1 / 2} B_{Y i-m j+1 / 2}-\Delta X_{j-1 / 2} B_{Y i-m j-1 / 2} .
\end{gathered}
$$

Using the divergence-free property of the magnetic field, the generic term of the sum of Eq. (105) can be recast as $\Delta Y\left(B_{x i-m-1 / 2}-B_{x i-m+1 / 2}\right)$. We therefore have:

$$
\frac{B_{X i-1 / 2}^{n+1}-B_{X i-1 / 2}^{n}}{\Delta t}=\frac{v_{j+1 / 2}^{0}}{\Delta X_{j+1 / 2} N}\left(B_{X i-N-1 / 2}^{n}-B_{X i-1 / 2}^{n}\right),
$$

which yields, since $v_{j+1 / 2}^{0} \Delta t=N \Delta X_{j+1 / 2}$ :

$$
B_{X i-1 / 2}^{n+1}=B_{X i-N-1 / 2}^{n} .
$$

Eqs. (103) and (107) show that in the special case under consideration the constrained transport using the upstream average electric field has characteristics similar to those of orbital advection in the hydrodynamical case: it merely amounts to a circular permutation of the cell values, with no numerical diffusivity, and no impact on the Courant condition. Instead of being hard coded as a shift as in the hydrodynamical case, however, here we require the evaluation of the upstream averages. Most of their terms cancel out, which de facto amounts to a shift to machine accuracy. In the more general case with shear and when the upstream arc does not represent an integer number of cells, the electromotive force on the residual arc is evaluated using a piecewise parabolic interpolation of the electric field ${ }^{15}$. The orbital advection of the magnetic field corresponds to cell 11 of Fig. 2 .

\subsection{Orbital integrator for planets}

Like its ancestor FARGO, the FARGO3D code features the possibility to simulate an arbitrary number of point-like masses around a central mass, which interact with the gas. To evolve planetary systems, we use the fifth order Cash-Karp method (Cash \& Karp 1990), a Runge-Kutta method with a fixed time step, here governed by the CFL condition. As the time step is decoupled from the interaction between bodies, this method does not ensure a good solution for close encounters. However, it is good enough for planetary systems in which planets do not suffer close encounters. Should more accuracy be needed at some point, it is easy to sub-cycle the planetary integration with an adaptive time step decoupled from the CFL condition to advance the planetary system with a high level of precision. We also mention that our N-body 
module is, like in the FARGO code, well decoupled from the rest of the code and easy to substitute, should one need a more sophisticated integrator. This has been for instance the approach of Morbidelli \& Nesvorny (2012), who implemented the SYMBA solver (Duncan et al. 1998) in the FARGO code in order to detect close encounters and collisions.

For the sake of completeness, we lay down here the algorithm used. The differential equation reads formally:

$$
\frac{d \vec{y}}{d t}=\vec{f}(t, \vec{y})
$$

where $\vec{y}$ is the $6 N$ vector component of the positions and velocities of the $N$ planets. The approximate solution given by the Cash-Karp method is:

$$
\vec{y}^{n+1}=\vec{y}^{n}+h \sum_{i}^{6} b_{i} \overrightarrow{k_{i}}
$$

with $\overrightarrow{k_{i}}=\vec{f}\left(t_{n}+c_{i} h, \vec{y}^{n}+h \sum_{j=1}^{6} a_{i j} \overrightarrow{k_{j}}\right)$, where the coefficients are given by the standard Butcher tableau (Tab. 3 .

The force exerted by the gas onto the planets is evaluated only once per hydrodynamical time $\operatorname{step}^{16}$, and used to update the planetary velocities. It is evaluated in either of two manners:

- by direct summation of the force exerted by all the cells,

- or by removing, prior to this summation, the axisymmetric part of the gas density to the content of all cells ${ }^{17}$. Since an axisymmetric disk cannot exert a torque on an embedded, coplanar planet, this does not alter the tidal torque exerted on the planet. An axisymmetric disk does however exert a radial force, on the planets and on itself. Since we do not take selfgravitation into account, the disk and the planets orbit in effectively different potentials, which leads to a spurious shift of resonances in the disk (Baruteau \& Masset 2008), which can severely bias the rate of change of orbital elements. A workaround to this issue without resorting to self-gravitational, expensive calculations, can consist in removing the axisymmetric component of the disk mass prior to the force evaluation.

The update of the planetary positions and velocities is performed in cell $5 \mathrm{a}$ of Fig. 2

\section{IMPLEMENTATION CONSIDERATIONS}

\subsection{General considerations}

FARGO3D is entirely written in $\mathrm{C}^{18}$, and parallelized using MPI (Message Passing Interface) and a slab domain decomposition in the $Y$ and $Z$ directions only (we have chosen to have the full extent of the $X$ direction, along which orbital advection is performed, on a unique processing element). As can be seen in Tab. 11. the mesh is therefore split radially and vertically in cylindrical coordinates, and in radius and colatitude in spherical coordinates. As in all grid based codes, the meshes are surrounded by several extra layers of cells, usually called buffer zones or ghost zones, which are used either to specify the boundary conditions, or to receive the values of neighboring meshes upon an MPI communication. The number of extra layers depends both on the problem at hand and the frequency with which communications are performed (i.e. how many times per time step). There is a trade off between the number of ghost layers and the frequency: the more frequent the communications, the thinner the ghost layers. In our implementation we perform communications only twice per time step (corresponding to cells 2 and 9 of Fig. 2), and we have three layers of ghost zones ${ }^{19}$. As this number also depends on the complexity of the problem, it may need an increase when new physics is included (such as thermal diffusivity, or any other new source sub step in which the new value of a zone depends on its neighbors). The correct number of ghost layers should be determined via an indiscernibility test: it is the minimum number of ghost layers that are required to yield an outcome strictly independent of the number of processors on which the test is spawn.

\subsection{Building for GPUs}

In order to avoid programming manually in CUDA (which is tedious and error prone) and in order to keep the development as simple as that of a code only meant to run on CPU, we have chosen to convert automatically expensive CPU routines to their CUDA counterpart. The computational cost comes from a particular kind of routines, that we dub mesh functions. A mesh function is a function that takes certain inputs such as scalar values, geometrical quantities and meshes (data cubes), then processes them using nested loops over the mesh in order to update a data cube. They correspond to the various substeps of the numerical algorithm. For instance, a mesh function is used to fill the pressure array, from the internal energy array. Another one is used to update the array of velocity in the $X$-direction from the pressure and potential gradients. Still another one is used to divide an array by another array, cell by cell (this is required by the consistent transport mentioned at the end of section 3.4p.

The general form of this kind of function is:

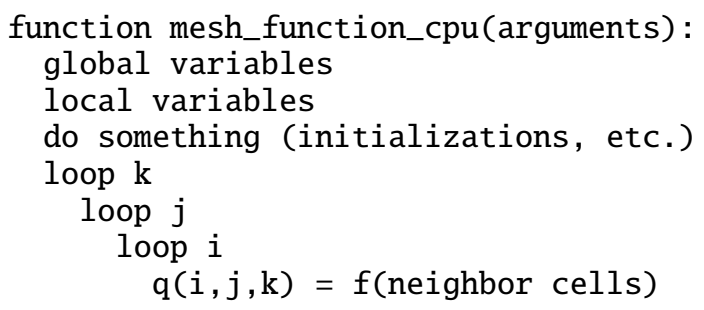

do something (e.g. reductions, etc.) end function

It is possible to build a few set of rules to transform this pseudo-language to any computational language, when the transformation rule for each specific part is defined. In order to transform this piece of pseudo code to CUDA, which generally has another memory space, it is also necessary to deal with transfers between host (CPU) and device (GPU) memories. Dealing manually with such transfers can quickly turn out to be a logistic nightmare, especially during the early phases of development, when some mesh functions are already running on the device while others are kept on the host. For this purpose, we have developed two commands, INPUT () and OUTPUT (), which automatically deal with data transfers between host and device: each mesh structure has two flags which indicate whether the data is up-to-date on the host and device memories. INPUT() uses this information to trigger a data transfer whenever required, while OUTPUT() resets both flags, depending on were the mesh function is 
block runs within one of the many streaming multiprocessors that exist on the GPU. The total number of threads thus spawned must be at least equal to the number of cells in our computational mesh, so that one thread updates the content of one cell. The division of the grid in blocks, and of the blocks in threads, must be done in an efficient way: if one chooses a small number of large blocks, the register pressure in each block may significantly degrade the performance. If, on the other hand, one chooses a large number of small blocks, the streaming multiprocessors may be underused when running these small blocks. For each setup we choose a characteristic block size, so that all CUDA kernels are launched with blocks of this size by default. However, we have also implemented an option in our parser which produces a code that wraps a given kernel invocation in a loop that explores all the possible block sizes and remember the size that yields the shortest execution time. By executing this search prior to a large run, we ensure that each kernel is launched with the best possible block size. This feature allows us to gain a further $10-20 \%$ speed up with respect to the unique default size. We emphasize that each of our mesh functions performs simple operations, and are designed to avoid race conditions. Should a race condition appear in one of them, we would have to split it into more elementary mesh functions. Therefore, the core part of each mesh function has exactly same structure in the CPU version (in that case it is the part executed within the nested loops) and in the GPU version (in that case it is the action executed by each thread upon completion of the indices' algebra). The only difference between these two versions is the algebra on array offsets: data are contiguous in the CPU memory, while they have some extra padding on the GPU to preserve the alignment of each row. Our conversion process is therefore simple and systematic, hence the performance achieved on the GPU (measured in terms of speed up factor with respect to the CPU version) does not vary significantly from kernel to kernel.

The parsing and production of CUDA code from the $\mathrm{C}$ code is done on the fly during the compilation of the code. The conversion process is guided by comments in the $\mathrm{C}$ code that follow strict syntax rules, which allow to separate the different parts of the mesh function (variables with a scope limited to the function, or internal variables; variables that must be passed as argument to the kernel, or external variables; core code of the mesh function, pre- and post-operations, etc.) Note that not all computationally expensive functions are mesh functions, in which one or several data cubes are updated as function of local data (values of data cubes in same cell or neighboring cells). This, in particular, is the case of reductions, in which one single value is obtained as a function of all cell values. Diagnostic functions, which integrate a value over the whole mesh (such as the mass or angular momentum), or the routine that uses the Courant condition (see section 3.5) to evaluate the maximal affordable time step, correspond to reductions (respectively with the sum and min operations). Reductions have been implemented once for all in the code ${ }^{21}$, following the efficient scheme of Harris (2007).

We typically achieve a $40 \times$ speed up in the execution on one GPU (with ECC activated) compared to one CPU of similar generation ${ }^{22}$ (e.g. between an Intel $^{\circledR}$ Core $^{\mathrm{TM}} \mathrm{i} 7950$ at 3.07 $\mathrm{GHz}$ and a Tesla C2050, or between an Intel ${ }^{\circledR}$ Xeon ${ }^{\circledR}$ E52609v2 at $2.5 \mathrm{GHz}$ and a Kepler 20), although this ratio depends on the problem and on the mesh size (larger speedups are obtained with larger meshes). The error control is natu- rally activated on the GPUs in these tests.

Table 4 presents the computational throughput and memory footprint of different setups. Tests $a, b_{1}$ and $b_{2}$ correspond to two-dimensional setups, while tests $c$ and $d$ correspond to three-dimensional setups. When MHD is included, all three directions are calculated and ghost zones are always added to the mesh in $Y$ and $Z$. The 2D Cartesian MHD tests $b_{1}$ and $b_{2}$, performed respectively in $Y Z$ and $X Y$, have very different memory footprints. The $Y Z$ test has an active mesh size (respectively in $X, Y$, and $Z$ ) of $1 \times 1024 \times 1024$, and the augmented mesh (including the ghost zones) has size $1 \times 1030 \times 1030$. Similarly, the $X Y$ set has an active mesh of size $1024 \times 1024 \times 1$, the augmented mesh has size $1024 \times 1030 \times 7$, hence an approximately 7 -fold larger memory footprint. Since calculations occur in the ghost zones, and since our computational throughput is evaluated using exclusively the cells of the active mesh, there is an apparent drop, typically by a factor of 7 , of the computational throughput of test $b_{2}$. The low throughput measured for test $b_{1}$ has a different origin: here the active and augmented meshes have sensibly same size. In our implementation, reductions are firstly performed on the GPU in the $X$-direction, and completed in the $Y$ and $Z$ direction on the CPU (the cost of these last two steps being negligible compared to the first one if there are many cells in $X$ ). Here, however, we only have one cell in the $X$ direction: the reduction (which corresponds to the search of the minimal timestep limit over the mesh) can be regarded as performed only on the CPU, which constitutes a bottleneck. This is the only bottleneck in our present implementation, and it occurs for setups which are not among the primary goals of the code.

Tab. 4 can also be used to estimate the maximal size of a simulation for a given platform. The memory footprint does depend on the problem dimensionality, on whether the equation of energy is solved, and on whether MHD is included, but it does not depend on the mesh geometry. We can for instance estimate that on a K20x device with $6 \mathrm{~Gb}$ of RAM, we could run a Cartesian three-dimensional isothermal HD simulation on a cube of size $\sim\left(6 \cdot 10^{9} / 168\right)^{1 / 3} \approx 329$ cells. This is naturally an upper limit, since some memory space must be reserved for auxiliary two- and one-dimensional arrays. Assuming arbitrarily that about $10 \%$ of the card memory should be reserved for overhead, we find that a simulation of size $320^{3}$ could fit on one K20x.

\subsection{MPI and GPUs}

Our implementation makes use of the peer-to-peer and universal virtual addressing features of CUDA, available since version 4.0, which allow to send data directly from one GPU to another one, thereby reducing the cost of inter-GPU MPI communications. This feature is requested at build time ${ }^{23}$, and can be disabled to fall back on traditional MPI communications between CPUs (accompanied with CPU-GPU data transfer), should peer-to-peer communications be not supported on a given platform. We note that the typical speed up ratio between a CPU core and a GPU is about 40, which is of the same order of magnitude as the bandwidth ratio between a gigabit and InfiniBand network. The scaling performance of a parallel GPU code on an InfiniBand cluster should therefore be similar to the scaling efficiency of its CPU version on a gigabit cluster with one core per nod ${ }^{2}$. We show in Fig. 4

2 Although a GPU cluster can have several GPUs per node, the bandwidth of the bus transited by data in intranode communications has same order of 


\begin{tabular}{lcc} 
Setup & Computational throughput (Mcell/s) & Memory footprint (bytes/cell) \\
\hline$a$ & 36.2 & 138 \\
\hline$b_{1}$ & 3.88 & 280 \\
$b_{2}$ & 2.44 & 2060 \\
\hline$c$ & 20.4 & 168 \\
\hline$d$ & 22.6 & 200
\end{tabular}

TABLE 4

COMPUTATIONAL THROUGHPUT AND MEMORY FOOTPRINT FOR DIFFERENT SETUPS. SETUP $a$ : HD ONLY, LOCALLY ISOTHERMAL, 2D (AZIMUTH AND RADIAL) IN CYLINDRICAL

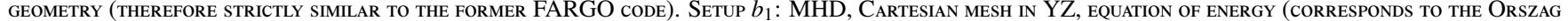

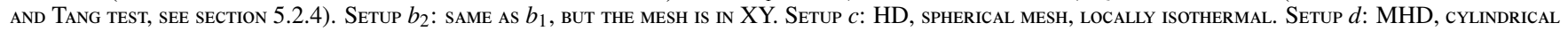
MESH, LOCALLY ISOTHERMAL (CORRESPONDS TO THE UNSTRATIFIED MRI TEST PRESENTED IN SECTION 6.1.2. THE LOW PERFORMANCE FOR THE SETUP $b_{1}$ IS DUE TO THE FACT THAT REDUCTIONS IN YZ ARE PERFORMED ON THE CPU (ONLY REDUCTIONS ALONG THE X AXIS ARE PERFORMED ON THE GPU). THE LOW PERFORMANCE FOR THE SETUP $b_{2}$ IS DUE TO THE FACT THAT THE XY MESH IS SLICED IN BETWEEN THREE LAYERS OF GHOST CELLS IN Z ON EACH SIDE (MHD SETUPS REQUIRE ALL THREE COORDINATES, REGARDLESS OF THE MESH SIZE). ALL TESTS HAVE BEEN PERFORMED ON ONE NVIDIA's K20 GRAPHICS CARD IN DOUBLE PRECISION. Note: all throughputs on the GPU have been measured with ECC on.

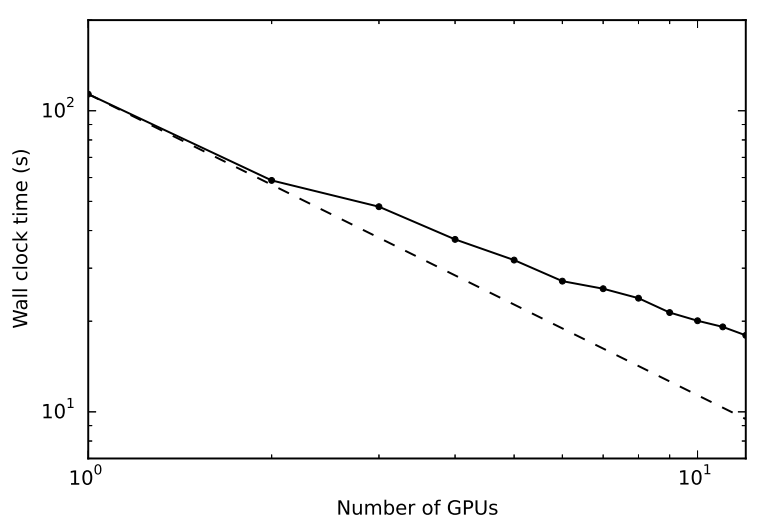

FIG. 4.- Wall clock time as a function of the number of GPUs, on a cluster of K20 GPUs, for a problem of given size (strong scaling test). The dots represent the results of measurements. The dashed line shows the ideal case obtained by extrapolating the time measured when the test is run on one GPU only.

the results of a strong scaling test of FARGO3D on a cluster of Kepler 20 graphics card. The cluster has four such cards per nodes, and the nodes are interconnected with an FDR InfiniBand network at $56 \mathrm{~Gb} / \mathrm{s}$. The setup considered in this test is irrelevant for the purpose of this section, and it suffices to know that the mesh considered has size $N_{x}=1300, N_{y}=435$ and $N_{z}=43$ (so that the problem just fits in the RAM of one graphics card).

\section{TESTS}

We present below a series of tests which all have been published elsewhere, for comparison purposes. Section 5.1 presents the purely hydrodynamical tests, while section 5.2 presents MHD tests. Within each section, the tests are broadly organized by increasing dimensionality of the setup.

\subsection{Hydrodynamical tests \\ 5.1.1. Sod shock-tube test}

The Sod shock tube test consists of a one-dimensional Riemann problem, in which a discontinuity (between two uniform states) is set up in the initial conditions. This standard hydrodynamical test has an analytic solution. Our domain is $0 \leq z \leq 10$, and the boundary between the two states $(-\&$ $+)$ is at $z=5$. We set $\rho^{-}=P^{-}=1$, while the right state has $\rho^{+}=0.125$ and $P^{+}=0.1$. The initial velocity is set everywhere to 0 . We use an adiabatic equation of state with a specific heats ratio $\gamma=1.4$. Figure (5) shows the result of

magnitude than the bandwidth of InfiniBand networks.
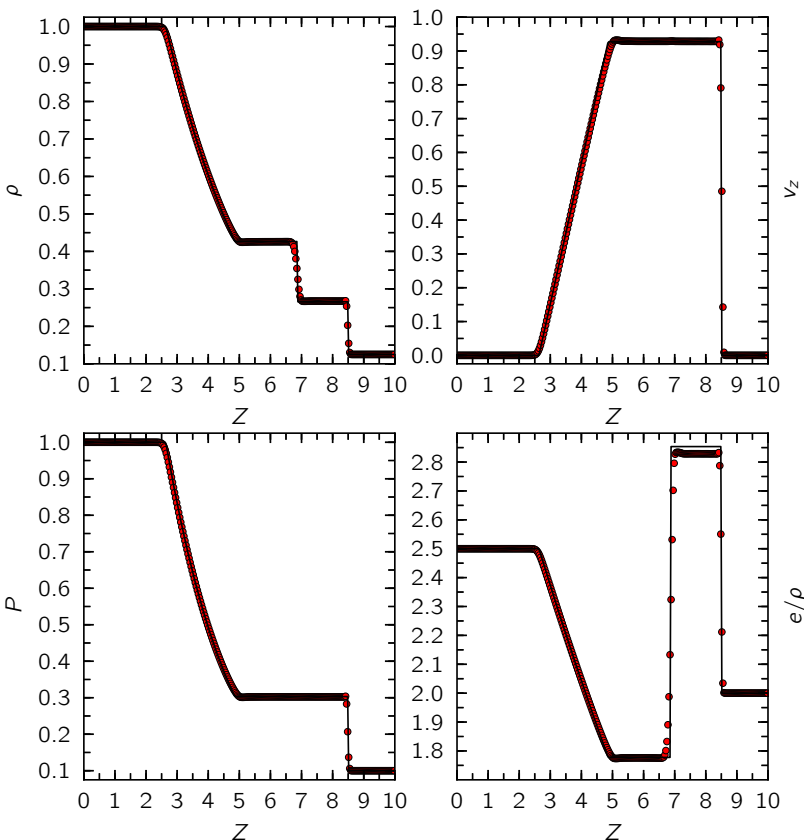

FIG. 5.- Sod shock tube test, with 300 grid cells, at $t=2$. The shock (at $z \approx 8.5$ ) is spread over $2-3$ zones, while the contact discontinuity (at $z \approx 6.9$ ) is spread over $7-8$ zones. There is a hardly noticeable offset of $\delta \rho / \rho \sim 10^{-2}$ between the numerical solution (red dots) and the exact one (solid black curve), between the contact discontinuity $(z \sim 7)$ and the shock $(z \sim 8.5)$. The impact of this offset is readily apparent on the right bottom plot, because of the reduced range of the $y$-axis.

this test, compared to the analytical solution. For this test we use 300 cells and van Leer's slope limiter. Despite a small deviation in the specific energy value, the results are in good agreement with the exact solution.

\subsubsection{Viscous spread of a gaseous ring}

One test to verify the validity of the viscous module is the gas ring diffusion problem (see Speith \& Riffert 1999). An axisymmetric thin disk around a central star experiences a radial drift as a consequence of viscous stresses. Integrating the hydrodynamical equations in $Z$ and neglecting pressure forces, the evolution for the density is governed by:

$$
\frac{\partial \Sigma}{\partial t}=\frac{3}{r} \frac{\partial}{\partial r}\left[\sqrt{r} \frac{\partial}{\partial r}(v \sqrt{r} \Sigma)\right] .
$$




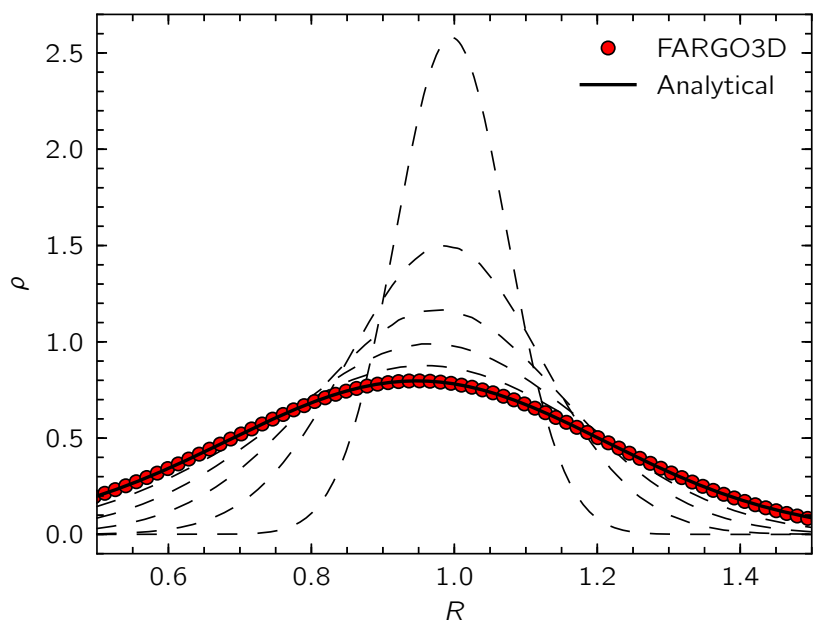

FIG. 6.- Viscous ring test. Dashed lines correspond to the numerical results for times $t=100,300,500,700,900$. The solid line corresponds to the analytical solution at time $t=1100$, while the red points show the numerical results at the same time.

Under this assumption, the azimuthal velocity is Keplerian, while the radial velocity is given by:

$$
r \Sigma v_{r}=-3 \sqrt{R} \frac{\partial}{\partial r}(v \sqrt{r} \Sigma) .
$$

It can be proven (Lynden-Bell \& Pringle 1974) that if the initial condition is that of an infinitely narrow axisymmetric ring of mass $M$ and radius $R_{0}$ :

$$
\Sigma(r, t=0)=\frac{M}{2 \pi R_{0}} \delta\left(r-R_{0}\right),
$$

and if the viscosity is a constant $v_{0}$, then the surface density evolves as:

$$
\Sigma(r, t)=\frac{M}{\pi R_{0}^{2}} \frac{1}{\tau u^{1 / 4}} I_{n}\left(\frac{2 u}{\tau}\right) \exp \left(-\frac{1+u^{2}}{\tau}\right),
$$

where $I_{n}$ is the modified Bessel function of the first kind, and the radial velocity evolves as:

$$
v_{r}(r, t)=\frac{6 v}{\tau}\left[r-\frac{I_{n-1}(2 u / \tau)}{I_{n}(2 u / \tau)}\right],
$$

where $u=r / R_{0}$ and $\tau=12 v_{0} t / R_{0}^{2}$.

In order to test the viscous module, we initialize an axisymmetric Keplerian ring, with an initial surface density profile and radial velocity given respectively by Eqs. (113) and (114), evaluated at $t=100$. Our constant viscosity is $v_{0}=10^{-5}$. The radial extent is $0.1 \leq r \leq 1.6$, and we use 512 cells evenly spaced, our mesh being cylindrical. Boundaries are set to the zero gradient condition for each primitive variable. In figure 6 we show the density at different times. There is a good agreement between the results and analytic expectations.

\subsection{Magnetohydrodynamics tests \\ 5.2.1. MHD Riemann problem}

The MHD Riemann problem, also known as the Brio \& $\mathrm{Wu}(1988)$ test, is a standard Sod shock tube test where the effects of a jump in the transverse magnetic field are considered. We perform this $1.5 \mathrm{D}$ test along the $z$ direction, using the same initial conditions and resolution as described by Stone \& Norman (1992b). The test consists in two initially
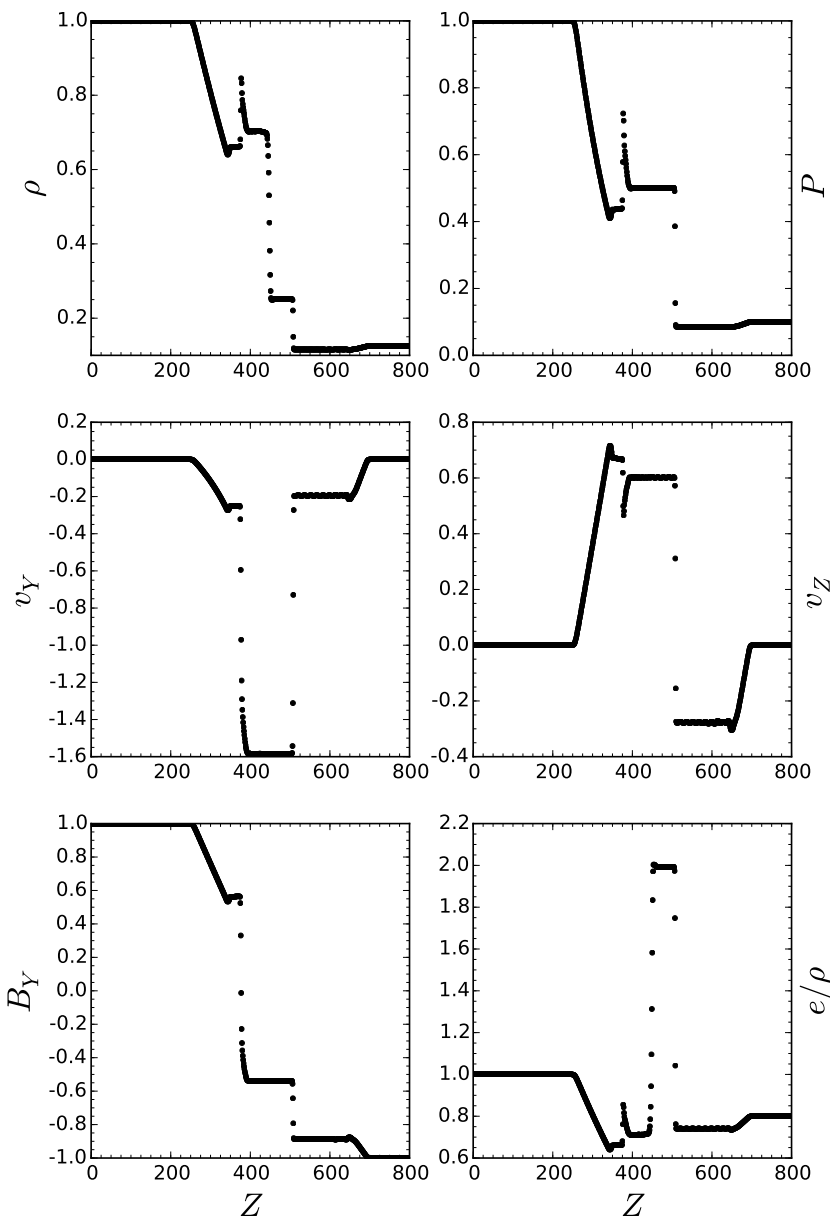

FIG. 7.- MHD Riemann problem, also known as the Brio \& Wu test. For the initial condition see the text.These results are to be compared with Fig. 6 of Stone \& Norman (1992b) and Fig 2. of Brio \& Wu (1988).

uniform states $(-\&+)$, separated by a discontinuity. The domain is $0 \leq z \leq 800$, where the discontinuity is located at $z=400$. At $t=0$ we initialize $P^{-}=1, P^{+}=0.1, \rho^{-}=1.0$, $\rho^{+}=0.1, B_{z}^{ \pm}=0.75$ while $B_{y}^{-}=1.0$ and $B_{y}^{+}=-1.0$. We use a ratio of specific heats $\gamma=2$ and 800 cells equally spaced over the domain. We use reflecting boundary conditions. Figure 7 shows different quantities at $t=80$.

Despite small oscillations in the velocity around $500 \leq z \leq$ 650 , we observe an overall good behavior for this test.

\subsubsection{Current Sheet Diffusion}

In order to test our implementation of the physical resistivity, we perform the following simple 1.5D test, in which a current sheet diffuses by Ohmic resistivity. The problem is formally defined as:

$$
B_{y}(z)=\left\{\begin{array}{cc}
B_{0} & z>0 \\
-B_{0} & z<0
\end{array}\right.
$$

and $B_{z}, v_{y}, v_{z}$ vanish over the whole domain. The resistivity is a constant $\eta_{0}$. We integrate the magnetic field passively, so that we discard the effect of the Lorentz force on the fluid evolution. We initially set a uniform pressure $P=1$, hence the velocity vanishes at all times. Since the velocity is zero, the induction equation reduces to a diffusion equation, and it 

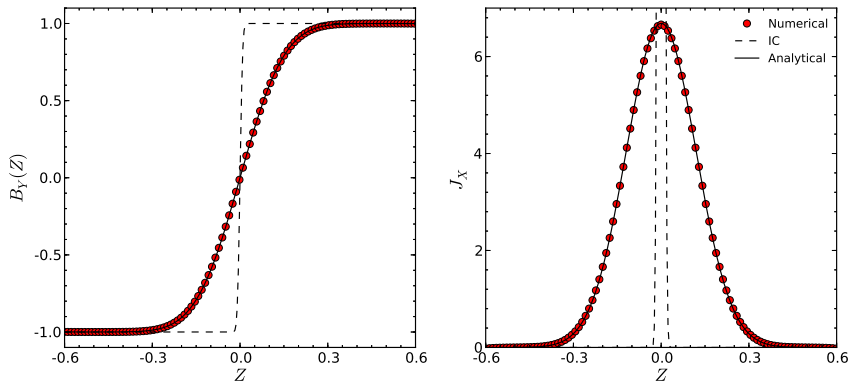

FIG. 8.- Current sheet diffusion test. The left panel shows the evolution of the magnetic field while the right panel shows the current. Dashed lines correspond to the initial conditions. Red points are the numerical solution at time $t=30 t_{0}$. The expected analytic solution, given by eq. 115, is shown with a solid line.

can easily be shown that the solution is given by:

$$
B_{y}(z, t)=B_{0} \operatorname{erf}\left(\frac{x}{\sqrt{4 \eta t}}\right) .
$$

Our domain is $-1 \leq z \leq 1$ and we use 512 cells evenly spaced. We have $B_{0}=1$ and $\eta=0.25$. In order to have a smooth behavior of the magnetic field, we use the profile given by Eq. (115) at $t_{0}=(5 \Delta z / 2)^{2}$ as initial condition for $B_{y}$, where $\Delta z$ is our the Z-size of a cell. This amounts to smoothing the current sheet over $\sim 10$ cells.

We plot the results in Figure 8, which shows a good agreement between analytics and numerics.

\subsubsection{MHD Rotor}

We perform the test suggested by Balsara \& Spicer (1999b), known as the MHD rotor test. It was designed for testing the propagation of strong torsional Alfvén's waves, which have implications in star formation problems. This test consists in having a dense, rapidly spinning cylinder in a light background fluid. The MHD Rotor test, by its geometry, is an excellent test to validate the implementation of the MHD solver in cylindrical coordinates. We have performed this test both in Cartesian and cylindrical coordinates.

In Cartesian coordinates, the test is defined in the $Y Z$ plane, with an initial state given by:

$$
\begin{aligned}
& \rho(y, z)=\left\{\begin{array}{cl}
1 & r<r_{0} \\
10 & r_{0} \leq r \leq r_{1} \\
1+9 f(r) & r_{1}<r<r_{2} \\
1 & r \geq r_{2}
\end{array}\right. \\
& v_{y}(y, z)=\left\{\begin{array}{cl}
0 & r<r_{0} \\
-v_{0}(z-0.5) / r_{1} & r_{0} \leq r \leq r_{1} \\
-f(r) v_{0}(z-0.5) / r_{1} & r_{1}<r<r_{2} \\
0 & r \geq r_{2}
\end{array}\right. \\
& v_{z}(y, z)=\left\{\begin{array}{cl}
0 & r<r_{0} \\
v_{0}(y-0.5) / r_{1} & r \leq r_{1} \\
f(r) v_{0}(y-0.5) / r_{1} & r_{1}<r<r_{2} \\
0 & r \geq r_{2}
\end{array}\right. \text {, }
\end{aligned}
$$

where $r=\sqrt{(y-1 / 2)^{2}+(z-1 / 2)^{2}}$ and the smoothing function $f$ is defined as $f(r)=\left(r_{2}-r\right) /\left(r_{2}-r_{1}\right)$. We set $r_{0}=0.05$, $r_{1}=0.1, r_{2}=0.115, v_{0}=2, \gamma=1.4$, a uniform pressure $P=1.0$, and an initially uniform magnetic field with
$B_{y}=5 / \sqrt{4 \pi}$ and $B_{z}=0$. We use $512^{2}$ cells uniformly distributed over a domain covering from $Y \in[-0.5,0.5]$ and $Z \in[-0.5,0.5]$.

For the cylindrical version of this test, the initial condition we adopt is the same for the density, we set $v_{r}=0$ everywhere and $v_{\phi}$ is given by:

$$
v_{\phi}(r)=\left\{\begin{array}{cl}
0 & r<r_{0} \\
v_{0} r / r_{1} & r \leq r_{1} \\
f(r) v_{0} r / r_{1} & r_{1}<r<r_{2} \\
0 & r \geq r_{2}
\end{array},\right.
$$

We use a grid with $\left(n_{r}, n_{\phi}\right)=(256,1024)$, the cells being uniformly distributed over a domain $r \in[0.01,0.5], \phi \in[0,2 \pi]$. Boundaries condition are reflective in both implementations and an adiabatic equation of state is used.

Results are shown in Figure 9 This figure can be compared to Fig. 2 of Balsara \& Spicer (1999b). Note that we have introduced a difference with respect to the standard MHD Rotor test: we preferred to put a hole in the inner region of the cylinder to alleviate possible issues arising from the inner boundary in the cylindrical setur ${ }^{3}$ Our results can be closely compared with the results displayed on the webpage of the FLASH code, which have the exact same conditions as ours, except that they consider a whole cylinder instead one with a hole in the center (http://flash.uchicago.edu, manual page of version 4.2, chapter VII, section 25.2.5).

\subsubsection{Orzag-Tang Vortex}

We perform the well known Orszag \& Tang (1979) magnetic vortex test. This test has become a standard test for MHD codes, and there is a lot of comparison material for it.

The problem is defined on the domain $0 \leq y \leq 1,0 \leq z \leq 1$, and the initial conditions are given by:

$$
\begin{aligned}
v_{y}(z) & =-\sin (2 \pi z) \\
v_{z}(z) & =\sin (2 \pi y) \\
B_{y} & =-B_{0} \sin (2 \pi z) \\
B_{z} & =B_{0} \sin (4 \pi y) \\
P & =5 /(12 \pi),
\end{aligned}
$$

with $B_{0}=(4 \pi)^{-1 / 2}$, the density and pressure being initially uniform with values respectively $25 /(36 \pi)$ and $5 /(12 \pi)$. We use a ratio of specific heats $\gamma=5 / 3$. The figure 10 can be directly compared with the figures 10 and 11 of Londrillo \& Del Zanna (2000).

\subsubsection{MHD Loop}

This two dimensional test is based on the advection of a magnetic closed loop. It consists in a weak magnetic loop located in a uniform density medium with a uniform velocity field. The test is performed in the limit of $\beta \gg 1$, where the magnetic pressure is negligible compared with thermal pressure. The initial conditions are similar to those of Gardiner \& Stone (2005) and Fromang et al. (2006). In order to have initially a divergence free magnetic field, we initialize the loop using the potential vector $\vec{A}$ :

$$
A_{x}(r)=\left\{\begin{array}{cl}
A_{0}(R-r) & \text { if } r<R \\
0 & \text { otherwise }
\end{array}\right.
$$

${ }^{3}$ Note that the results presented in this paper do not correspond to the initial conditions advertised by Balsara \& Spicer (1999a). 

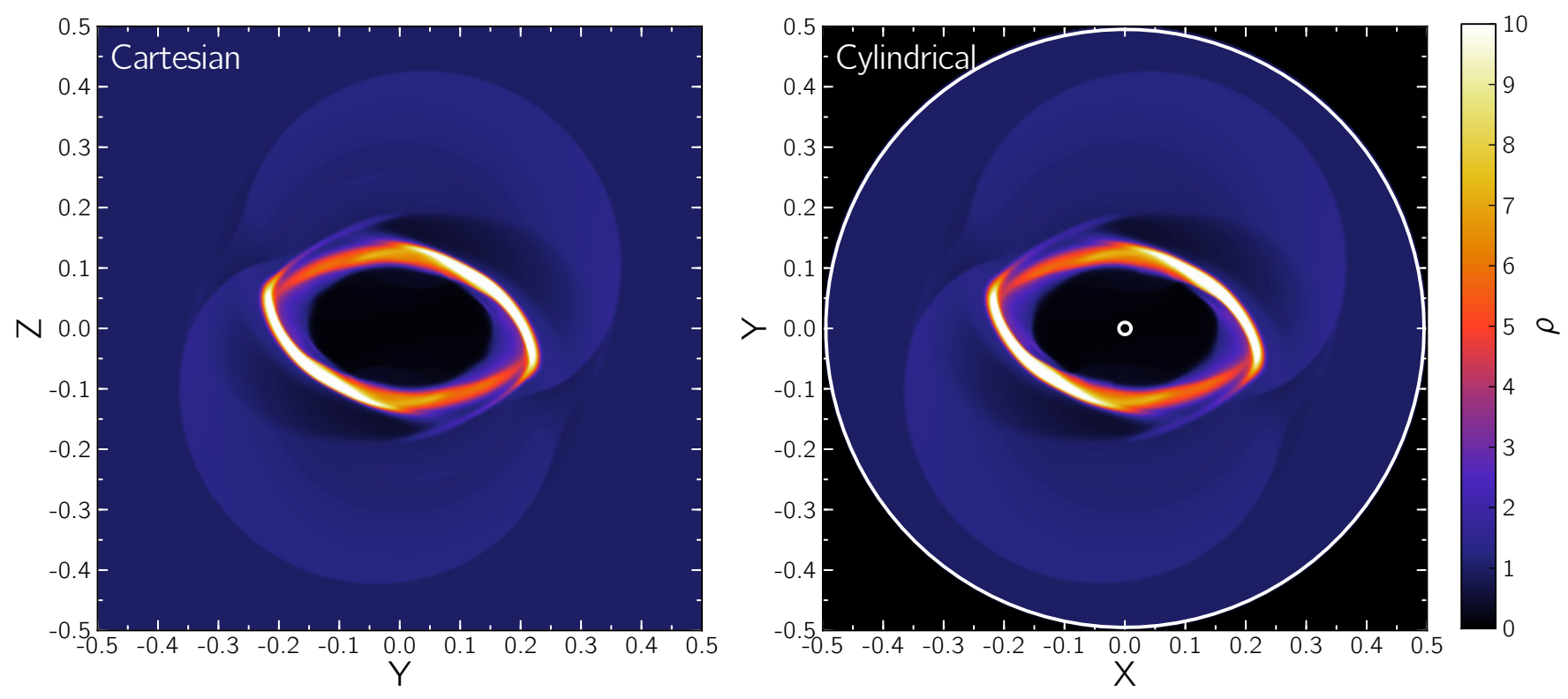

FIG. 9.- MHD ROTOR test results at $t=0.15$. In the left panel the Cartesian version of the test is shown. In the right panel, the cylindrical version is plotted, showing with white lines the boundaries of the cylindrical mesh. As long as the torsional waves launched by the central rotor do not reach the domain boundaries, as it is the case here, the agreement between both panels is remarkable.

with $A_{0}=10^{-3}, R=0.3$ and $r=\sqrt{y^{2}+z^{2}}$. We use a Cartesian mesh over the domain $-1 \leq y \leq 1,-0.5 \leq z \leq 0.5$. We use 128 cells in $y$ and 64 in $z$. Periodic boundary conditions are used in $y$ and $z$. We perform two simulations for $v_{y}=\sqrt{(5)} / 2, v_{z}=0$ and $v_{y}=1.0, v_{z}=0.5$, corresponding respectively to horizontal and diagonal advection. We show in Figure 11 the evolution of the mean magnetic energy of the mesh and the shape of the loop upon an advection over one full spatial period for the two cases. The decay of magnetic energy for the horizontal advection case should be compared to the curves given Fromang et al. (2006) in their Fig. 5. As these authors have twice a larger advection speed than ours, one should compare the magnetic energy at $t=2$ in our case $\left(E_{m} \approx 1.235 \cdot 10^{-7}\right)$ to theirs at $t=1$ (respectively $E_{m} \approx 1.225 \cdot 10^{-7}$ and $E_{m} \approx 1.19 \cdot 10^{-7}$ for the Roe Riemann solver and for the Lax-Friedrichs solver, so that on this specific problem the decay of magnetic energy that we get is similar to the one obtained by these authors with the Roe Riemann solver).

\subsubsection{Current Sheet}

In order to assess the numerical diffusivity of the MHD solver, we perform the current sheet test. It consists in a two dimensional problem in which discontinuities of magnetic field create current sheets. The details of the test have been designed by Gardiner \& Stone (2005), and this test has also been performed by Fromang et al. (2006). We do not include physical resistivity in this test, so that the evolution of the sheet is exclusively governed by numerical resistivity.

We use the exact same setup as Gardiner \& Stone (2005) and Fromang et al. (2006): our computational domain is $0 \leq$ $y \leq 2,0 \leq z \leq 2$ and the resolution is 256 in each direction. Initially, the density is $\rho=1$, the pressure $P=0.1$ and the magnetic field components $B_{x}=0$ and $B_{y}=0$ everywhere, while $B_{z}$ is given by:

$$
B_{z}(y)= \begin{cases}+1 & \text { if }|y-1|<0.5 \\ -1 & \text { otherwise }\end{cases}
$$

The velocity is initialized as $v_{z}=0$ everywhere and $v_{y}=$ $v_{0} \sin (\pi z)$. We use $v_{0}=0.1$ and $\gamma=5 / 3$. In figure 12 we show the evolution of the magnetic field lines. We see a behavior very similar to that of Fromang et al. (2006). At larger time $(t>2.5)$, four islands remain along the location of each discontinuity, instead of two in the case of Gardiner \& Stone (2005). We also note that our scheme conserves the central symmetry with respect to the velocity nodes, as does the RAMSES code on this test (Fromang et al. 2006).

\section{DISCUSSION}

The tests presented in the previous section allow us to validate the implementation of the different part of the core algorithm of the FARGO3D code, in different geometries. We now turn to a discussion about some aspects of the code. Namely, in a first part, we assess the impact of orbital advection on the code properties for two setups which are of high interest for the scope of the code. Secondly, we address briefly the question of single versus double precision calculations, which is a recurrent question in high performance computing on GPUs.

\subsection{Impact of orbital advection on the code properties}

\subsubsection{Vortex in a two-dimensional Keplerian disk}

We reproduce here the test described in section 3.1 of Mignone et al. (2012). Our setup and Courant number are exactly the same as those used by Mignone et al. (2012) and our runs are performed for the same three resolutions as in that work. The setup consists of a two-dimensional disk orbiting a central potential $\Phi=-1 / r$, with uniform surface density $\Sigma \equiv 1$ and pressure $p \equiv 1 /(\gamma \mathcal{M}), \mathcal{M}$ being the Mach number of the Keplerian flow at $r_{0}=1$, chosen to be $\mathcal{M}=10$. The unperturbed flow is exactly Keplerian, since there is no pressure gradient. Superimposed on this flow, we impose a perturbation $\left(\delta v_{\phi}, \delta v_{r}\right)$ of velocity that has the form:

$$
\left(\begin{array}{l}
\delta v_{\phi} \\
\delta v_{r}
\end{array}\right)=K \exp \left(-\frac{x^{2}+y^{2}}{h^{2}}\right)\left(\begin{array}{cc}
-\sin \phi & \cos \phi \\
\cos \phi & \sin \phi
\end{array}\right)\left(\begin{array}{c}
-y \\
x
\end{array}\right),
$$

where $h=c_{s}\left(r_{0}\right) / 2 \Omega\left(r_{0}\right)$ is the vortex size, $K=-1$ is the vortex amplitude, $x=r \cos \varphi-r_{0} \cos \varphi_{0}$ and $y=r \sin \varphi-r_{0} \sin \varphi_{0}$ 


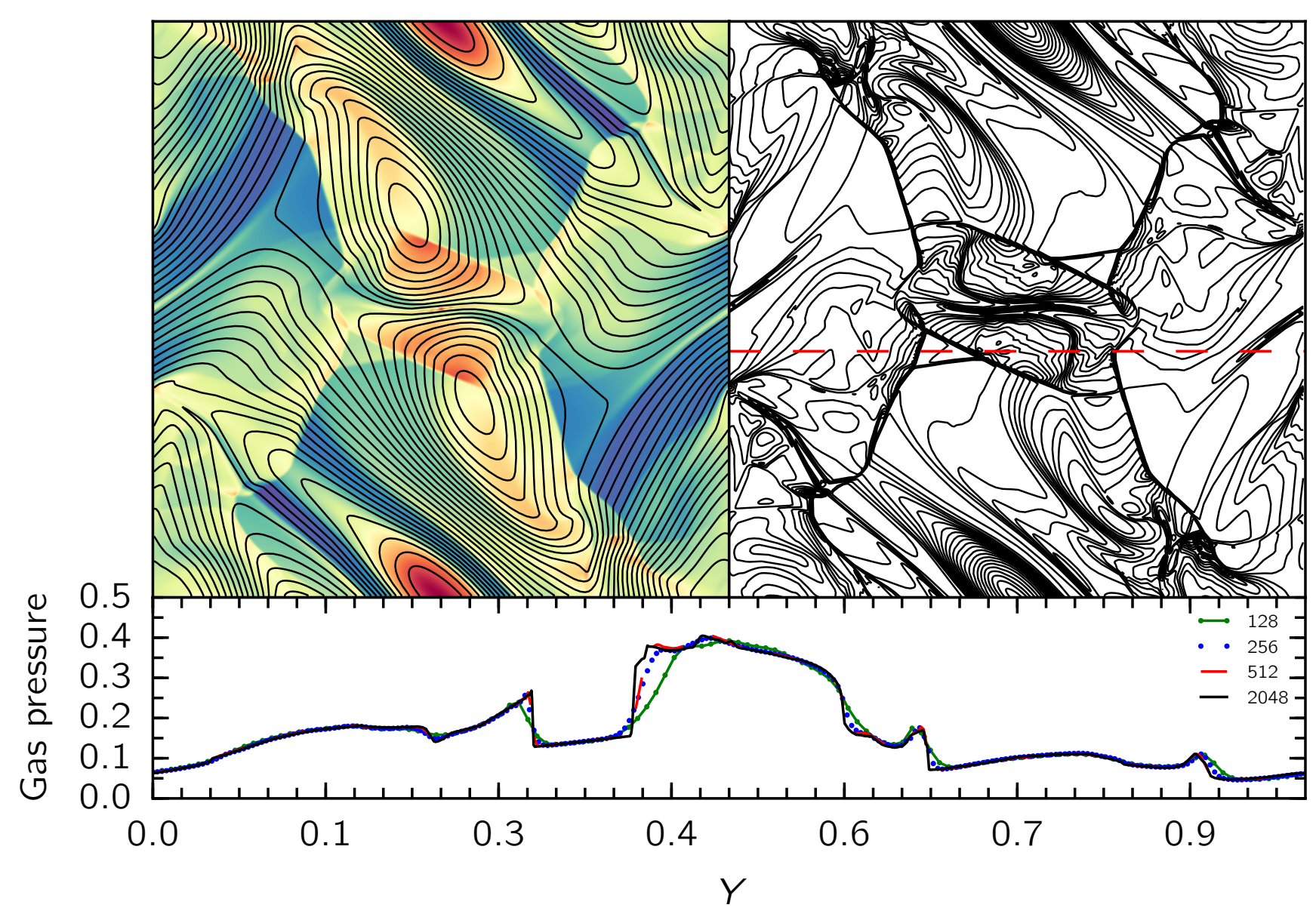

FIG. 10. - Orzag-Tang vortex test at $\mathrm{t}=0.5$. The upper left panel shows in gray scale the density map, with the magnetic field lines superimposed. The upper right panel shows the gas pressure contours. In the bottom panel we show a cut along the dashed line shown in the right panel ( $\mathrm{y}=0.4277)$. This cut is common in literature, and can be used for comparison purposes.

are the Cartesian coordinates measured in the frame centered on the vortex, which is located initially at $r_{0}$ and $\varphi_{0}=\pi / 4$. The results in Fig. 13 show that FARGO3D, like the PLUTO code, benefits significantly from orbital advection, as this dramatically improves the conservation of vortensity at all resolutions. Qualitatively, without orbital advection, vortensity conservation is slightly better, at a given resolution, with FARGO3D than with PLUTO, whereas the opposite holds when orbital advection is turned on.

\subsubsection{MRI in unstratified disks}

We present a calculation of the development of the magneto-rotational instability (MRI) in cylindrical coordinates with no vertical external force, with a setup similar to that of Baruteau et al. (2011). The mesh has a radial extent $1<r<8$, a vertical extent $-0.3<z<+0.3$, and covers half a disk in azimuth: $0<\varphi<\pi$. A locally isothermal equation of state is used (see eq. 6), in which we have everywhere $c_{s}(r)=0.1 v_{k}(r)$, where $c_{s}$ is the sound speed and $v_{k}(r)=\left(G M_{\star} / r\right)^{1 / 2}$ the Keplerian speed at radius $r$. Periodic boundary conditions are used in $z$. The density follows a power law of radius: $\rho \propto r^{-1 / 2}$ (the multiplicative constant of this law does not matter in an isothermal setup). The magnetic field is initially exclusively toroidal, and such the thermal to magnetic pressure ratio be uniform and equal to $\beta=50$. We introduce some noise on the radial and vertical components of the velocity, with an amplitude of $5 \%$ of the sound speed. We also follow a standard practice that consists in introducing resistive buffers in the inner part $(r=1$ to $r=2)$ and outer part ( $r=7$ and $r=8$ ) of the mesh. The resistivity scales linearly in these buffers, from zero at the frontier with the active disk to a maximal value at the mesh edge. This maximal value is $10^{-4}$ at the outer edge, and $2 \cdot 10^{-5}$ at the inner edge. Similarly to Baruteau et al. (2011), our mesh is rotating with an angular velocity $\Omega_{f}=r_{0}^{-3 / 2}$, which sets the corotation at $r_{0}=3$. Symmetric boundary conditions are applied in radius for the radial component of the EMF, whereas antisymmetric conditions are applied on the azimuthal and vertical components in order to conserve the magnetic flux to machine accuracy. We run two calculations, each over 300 orbits at the inner edge. One calculation uses orbital advection (see section 3.7), whereas the other does not. We refer to the latter as the standard calculation.

Fig. 14 shows the value of the $\alpha$ coefficient as a function of radius, time averaged between 120 and 300 orbits at the inner edge (over this time frame the turbulence appears to have reached a saturated state at all radii in both calculations), 


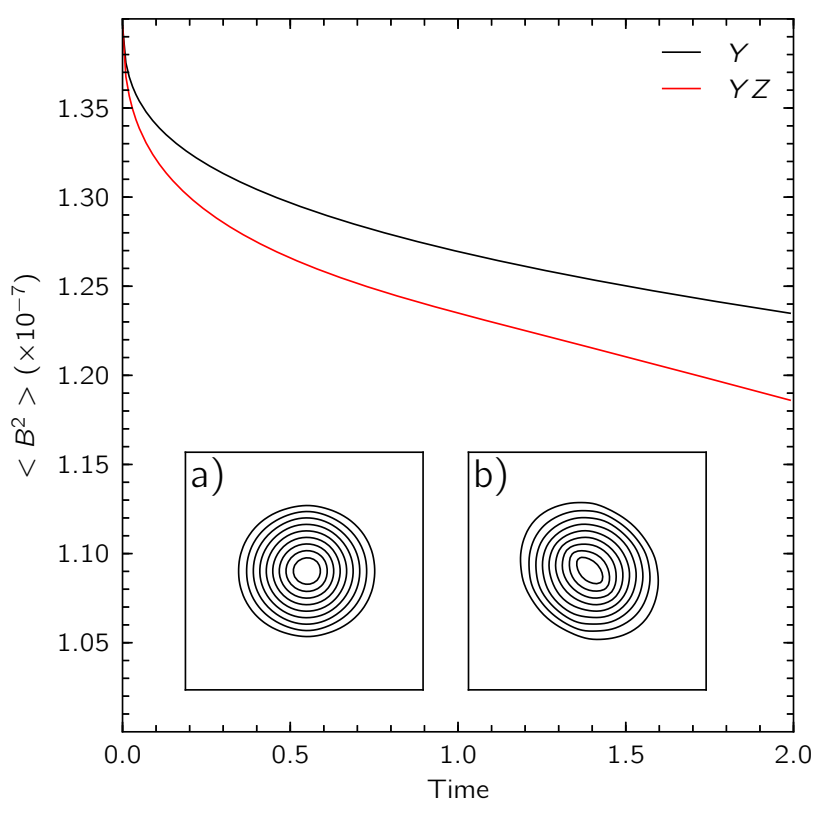

FIG. 11. - The main panel shows the time evolution of the mean magnetic energy in the magnetic loop advection test. We show in the inset panels the shape of the loops after a first travel over the mesh, for the horizontal advection case in panel $a$ and for the diagonal advection case in panel $b$.

where $\alpha$ is defined as:

$$
\alpha(r, t)=\alpha_{\text {Reynolds }}(r, t)+\alpha_{\text {Maxwell }}(r, t),
$$

with:

$$
\alpha_{\text {Reynolds }}(r, t)=\frac{\iint \rho \delta v_{r} \delta v_{\phi} d \phi d z}{c_{s}^{2}(r) \iint \rho d \phi d z},
$$

where $\delta v_{r}$ and $\delta v_{\phi}$ are the velocity fluctuations with respect to the mean flow at a given radius, and:

$$
\alpha_{\text {Maxwell }}(r, t)=-\frac{\iint B_{r} B_{\phi} d \phi d z}{\mu_{0} c_{s}^{2}(r) \iint \rho d \phi d z} .
$$

We recover the fact that the standard advection scheme is more diffusive away from corotation (Johnson et al.|2008) and consequently that the $\alpha$ coefficient is lower for the standard calculation than for the calculation with orbital advection, except at corotation $(r=3)$ where their values coincide.

Fig. 15. shows the temporal behavior of the $\alpha$ coefficient in both calculations, averaged from radius $r=2.1$ to $r=6.5$. As anticipated, its value levels off at a slightly smaller level in the standard case, which also reaches saturation a bit later than the calculation with orbital advection. Fig. 14 and 15 should be compared to Fig. 6 of Baruteau et al. (2011), in particular with the curve displaying NIRVANA's results, which were obtained for the same initial value of $\beta$. Our runs display a significantly larger plateau value of $\alpha$, but saturation is reached in a longer time. This might be due to the fact that no resistive buffers were used in the simulation with NIRVANA.

Fig. 16 shows the averaged value $\bar{\theta}_{B}$ of the tilt angle of the magnetic field, defined by Sorathia et al. (2012) or Mignone et al. (2012) as:

$$
\sin 2 \theta_{B}=\frac{\left|B_{\phi} B_{r}\right|}{P_{\mathrm{mag}}} .
$$

It converges towards a value comprised between $15^{\circ}$ and $16^{\circ}$ in the FARGO-MHD case, and towards a marginally lower value in the standard case. Again, the leveling off indicative of the saturation of turbulence is reached later in the standard case.

Fig. 17 shows the spectrum of the azimuthal component of the magnetic field, for the standard and orbital advection cases, in two rings: one sitting above corotation, and one located further out in the disk. These are obtained as:

$$
m B_{\phi}(m)=m \sum_{\gamma=0}^{N_{z}-1} \sum_{\beta=\beta_{\min }}^{\beta_{\max }}\left|\sum_{\alpha=0}^{N_{\phi}-1} B_{\alpha \beta \gamma} e^{i \frac{2 \pi}{N_{\phi}} \alpha m}\right|,
$$

where in the above expression we use $\alpha \beta \gamma$ indices instead of $i j k$ in order to avoid confusion with $\sqrt{-1}$, and where $\beta_{\min }$ and $\beta_{\max }$ are the indices of the edges of the ring over which the spectra are calculated. The spectra of both calculations are sensibly the same for the first ring (the orbital advection case showing nevertheless systematically more signal at higher azimuthal wave numbers), whereas the spectra of the outer ring show smaller resolved scales in the FARGO-MHD case, roughly by a factor of 2 , as noted by Mignone et al. (2012).

\subsection{Single or double precision?}

While calculations done on CPUs are most of the time done in double precision, the question of whether a given calculation should be performed in single or double precision arises frequently in the GPU HPC community. This, in part, is due to the limited amount of memory available on GPUs compared to their host counterparts. It is also due to the fact that the endeavor of coding for GPUs is reserved for very computationally demanding tasks, and working in single precision typically means an extra factor of two in computational throughput. There is no definite answer to that question: the choice of single versus double precision should be made on a case by case basis. We hereafter entertain two cases in the context of astrophysical disks, which we think represent two extreme cases. In one of them, the use of single precision floating point is obviously to be discouraged, while for the other case the use of single precision yields essentially same results as double point precision.

\subsubsection{Two dimensional laminar disk at high resolution}

In this case, we consider an Earth-mass planet embedded in a locally isothermal, inviscid disk with an initially uniform surface density, and a constant aspect ratio $h=0.05$. The mesh extends from $r=0.6 a$ to $1.4 a$, where $a$ is the planetary orbital radius. It has $N_{\phi}=4000$ cells in azimuth, and $N_{r}=$ 2000 cells in radius, which implies a radial resolution of $\Delta r=$ $4 \cdot 10^{-4} a$. The calculation is performed in the frame corotating with the planet. Fig 18 shows how the torque behaves in the single and double precision cases.

Some insight into the reasons for the behavior observed in the single precision case can be gained by evaluating the number of significant digits over which the vorticity is represented in the single precision case. The difference between the single and double precision values of the azimuthal velocity at $t=0$ shows typical r.m.s. fluctuations of the single precision value of $\sigma \sim 10^{-7}$.

The (vertical component of the) vorticity is obtained by deriving radially the azimuthal velocity (appropriately weighted to account for the cylindrical geometry). Since the velocity and its radial derivative are $O(1)$, we expect the vorticity, in the single precision case, to be accurate only to within 

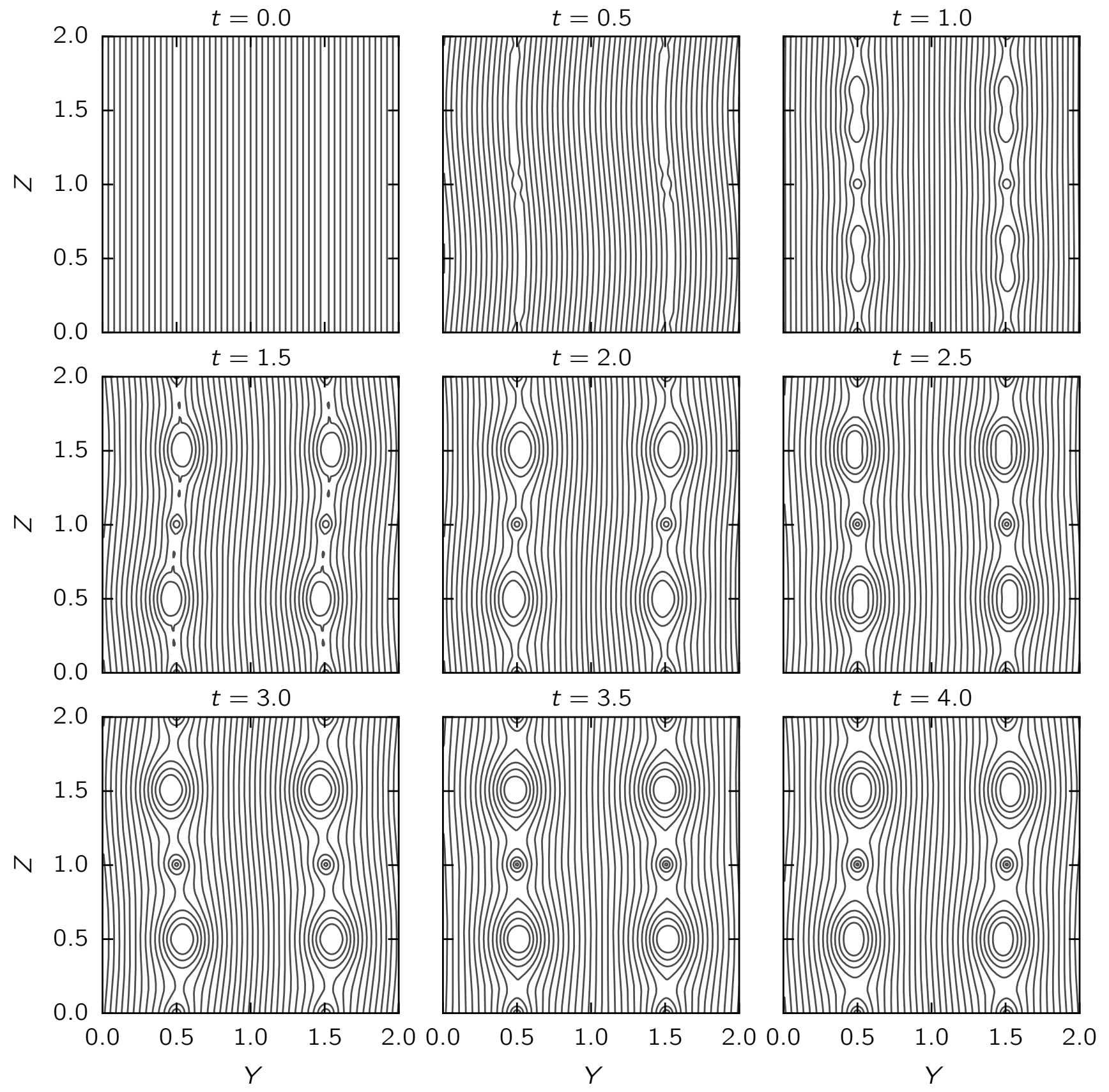

Fig. 12.- Time evolution of the magnetic field lines in the current sheet test of Gardiner \& Stone 2005). The box has $256^{2}$ cells. The times of the snapshots are shown on the top of each panel.

$\sim \sqrt{2} \sigma / \Delta r \sim 3.5 \cdot 10^{-4}$. This is precisely what we find on the difference of the single and double precision values of the vorticity. This typical error on the vorticity is comparable to its variation between successive radial bins $\Delta r\left|\partial_{R} \omega_{z}\right| \sim \Delta r$, since $\partial_{R} \omega_{z}=O(1)$. Consequently, the vorticity is not a monotonous function of radius in single precision, as we see in Fig. 19. and neither is the vortensity. Local extrema of vortensity are unstable to the Rossby wave instability (RWI, Lovelace et al. 1999, Li et al. 2000). We find that the computational domain is invaded by vortices after a few dynamical times in the single precision case (see Fig. 20). The acoustic wakes triggered by these vortices pass in the planet vicinity where their combined action yield the stochastic behavior of Fig. 18. Our interpreta- tion is that these vortices are triggered by the RWI which occurs at many radii in the single precision case, the breakdown of azimuthal symmetry being provided by the planet. We note that the problem may be worsened by our numerical algorithm: at each timestep, a new momentum and new density is inferred for each cell, the division of which yields the new velocity (this kind of procedure is commonplace in numerical hydrocodes). This may induce a random walk of the velocity with respect to the double precision value. We comment that adopting a significantly smaller resolution in single precision mitigates the problem exposed here (albeit this cannot be regarded as a solution or workaround), and that a similar issue should be expected in double precision calculations at 

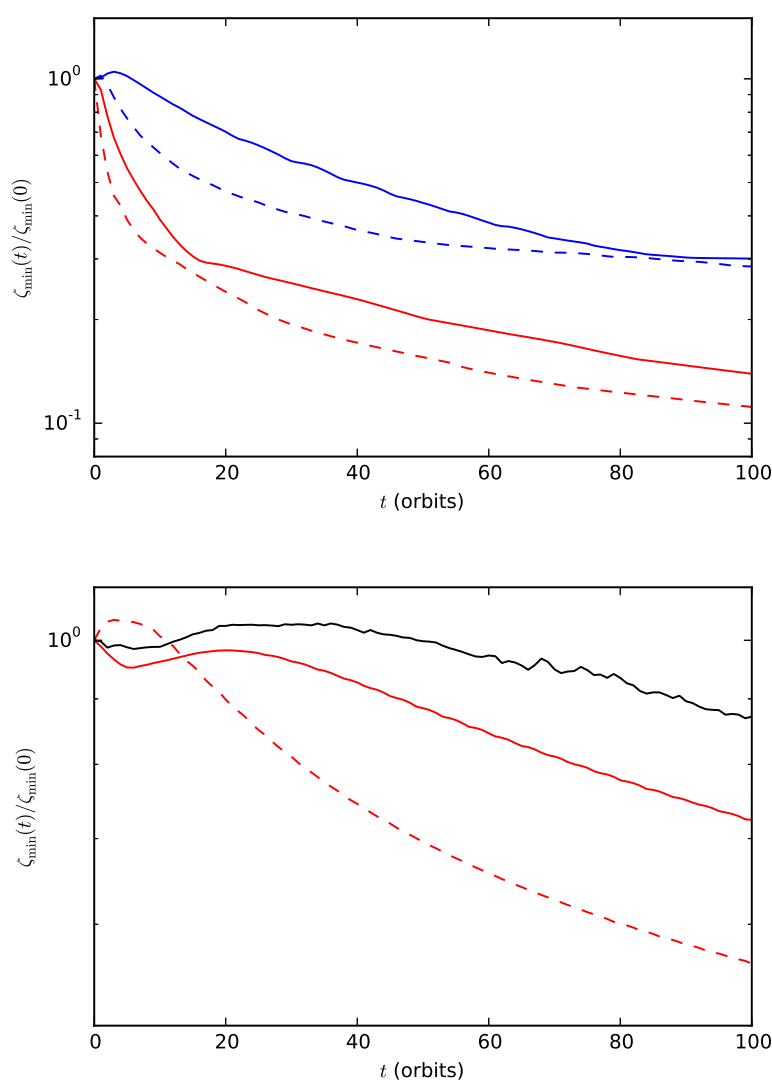

FIG. 13.- Left: decay of vortensity minimum as a function of time for resolution $1024 \times 256$ (red) and $2048 \times 512$ (blue). Solid lines show the results with orbital advection, and dashed lines without it. Right: results for the highest resolution case $(4096 \times 1024)$. The red curves show results for FARGO3D (with and without orbital advection) and the black curve shows the results for PLUTO with orbital advection (which we have reproduced using the version 4.0 of that code)

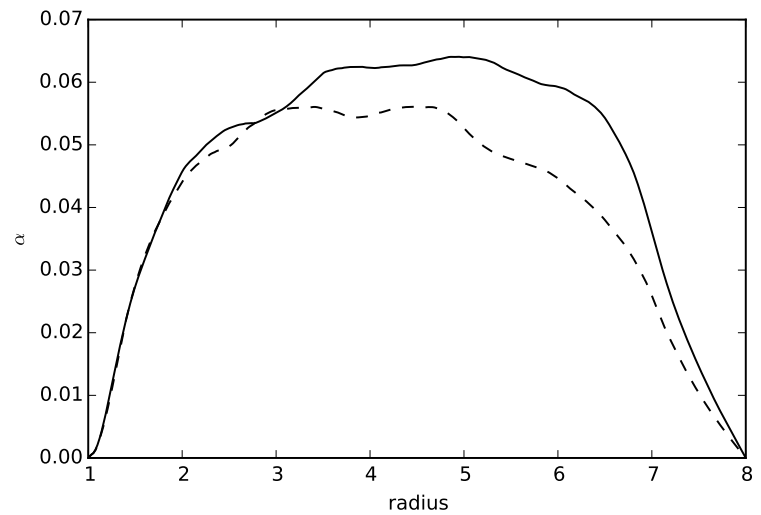

FIG. 14.- Value of $\alpha$ as a function of radius for the FARGO-MHD case (solid line) and standard case (dashed line). The drop near the edges is attributable to the resistive buffers.

much larger resolution (typically for $\Delta r \sim \sqrt{10^{-16}} a=10^{-8} a$, largely beyond the reach of present day platforms, at least over the time scale over which RWI sets in).

\subsubsection{Magnetorotational Instability in unstratified disks}

We consider again the setup of section 6.1.2, which we now run both in single and double precision. We compare

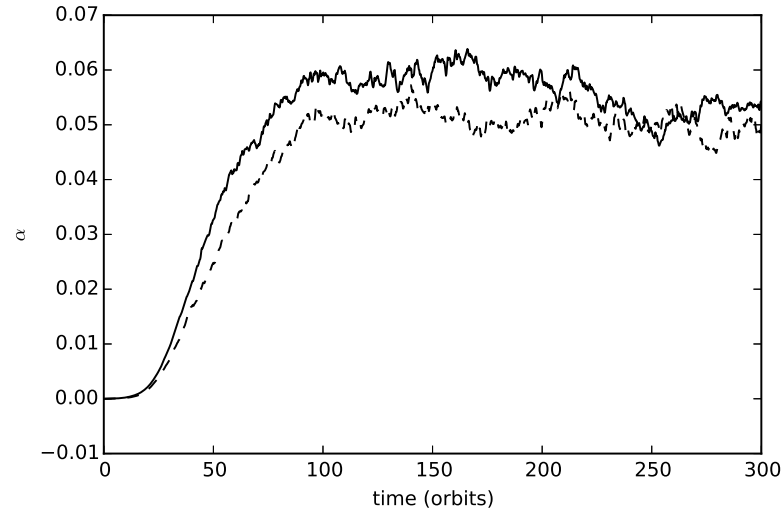

FIG. 15.- Value of $\alpha$ as a function of time for the FARGO-MHD (solid line) and standard (dashed line) cases.

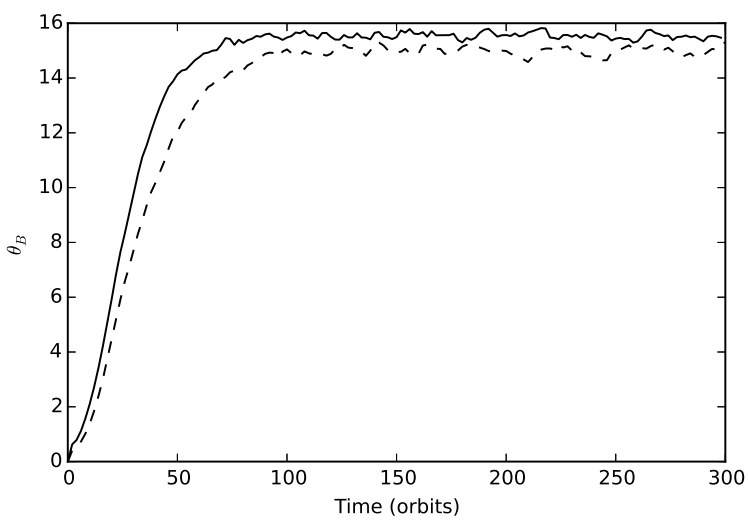

FIg. 16. - Value of the tilt angle of the magnetic field (in degrees) as a function of time for the FARGO-MHD (solid line) and for the standard (dashed line) cases.

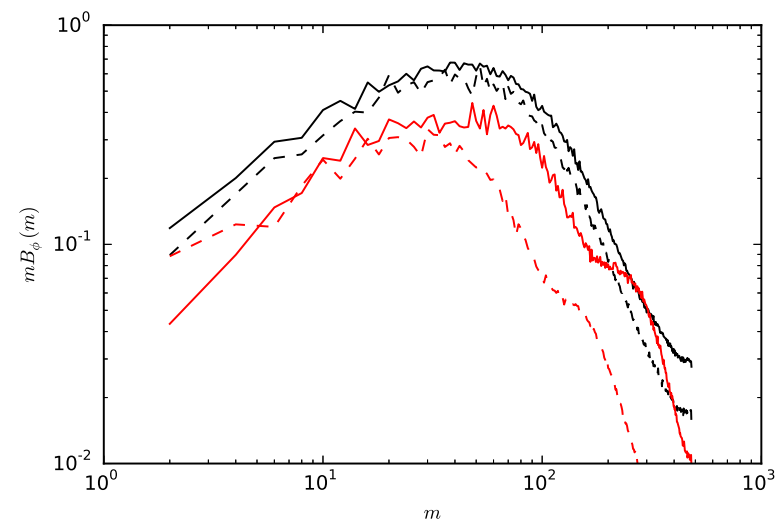

FIG. 17.- Spectrum of the azimuthal component of the magnetic field as a function of the azimuthal wave number. Solid lines refer to the FARGOMHD case, dashed lines to the standard case. The black curves correspond to a narrow ring encompassing corotation $(r=2.76$ to $r=3.24)$ whereas the red curves correspond to the outer ring $(r=6.00$ to $r=6.48)$. Both spectra are obtained at $t=100$ orbits at the inner edge. Note that our spectra are defined only for even values of $m$ since our azimuthal domain spans only $\pi$ radians. 


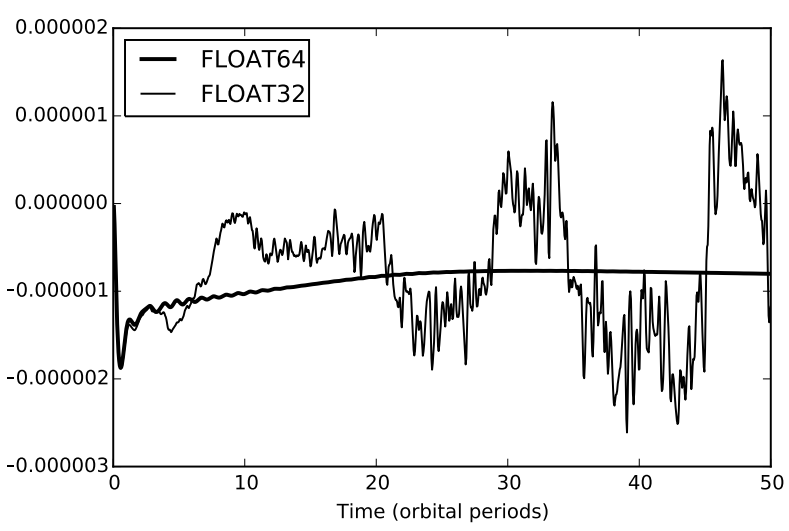

FIG. 18. - Specific torque exerted by the disk on the planet, as a function of the time elapsed after the planet insertion in the disk. The nearly horizontal, thick curve represents the torque obtained in the double precision calculation, while the thin, very time variable curve represents the torque in the single precision case. Apart from the floating point accuracy, the two setups are strictly identical. The torques begin to diverge after $3-4$ orbits.

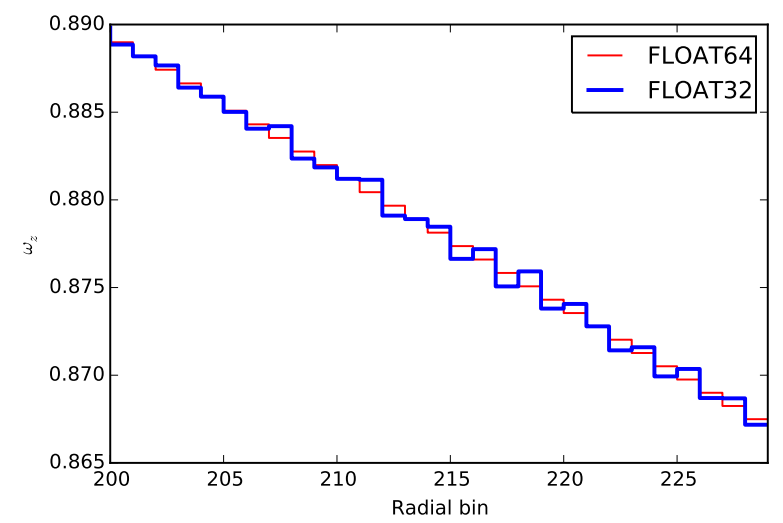

FIG. 19.- Close up of the vertical component of the vorticity as a function of radius, near the inner disk edge. The thin red line shows the double precision data, which is well behaved (the vorticity decays monotonically), and the thick blue line shows the single precision data, which shows many local maxima and minima.

in Fig. 21 the time averaged value of the $\alpha$ coefficient as a function of radius for the single and double precision calculations. In order to get a sense of the variation expected from one realization to another, we display for each case two calculations made with different random seeds. We see that the single precision case yields results are perfectly comparable to those obtained in double precision. Other diagnostics such as those shown in section 6.1.2 yield similar conclusions.

\section{PERSPECTIVES}

The development of an astrophysical code for fluid dynamics is generally an unending process in which new physics as well as minor improvements are constantly added. We draw below a list of some of our recently added features and ongoing projects, not publicly released yet, which will be presented in full detail elsewhere. Nonetheless, the features that are presented in this paper are the basic blocks of the code, and we intend to keep them unchanged for the foreseeable future. They constitute the bases that any user interested in working on the code should know.

Recent work has highlighted the importance of radiative transfer processes for the physics of protoplanetary disks

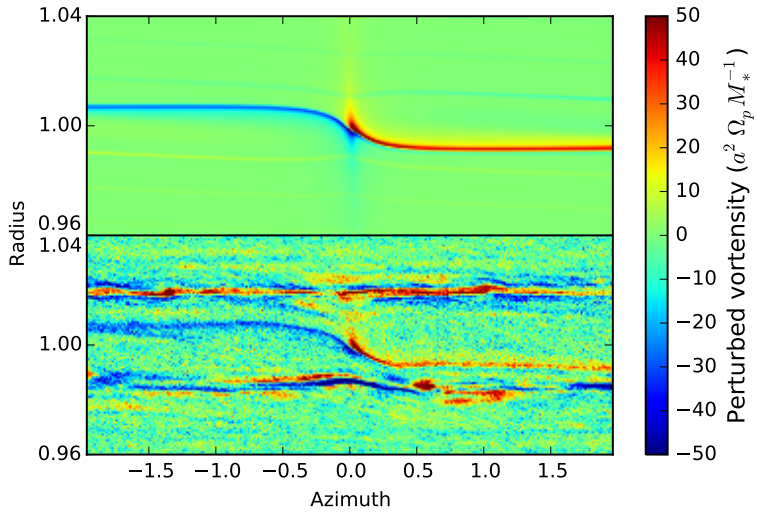

FIG. 20.- Perturbed vortensity distribution after 50 orbital periods, in the double precision case (top) and single precision case (bottom). The blue and red stripes originating near the planet are physical, and come from vortensity's advection in the planetary horseshoe region on the one hand, and the driving of vortensity arising from the radial temperature gradient on the other hand (Casoli \& Masset 2009). The single precision case shows significant noise in the data, owing to the small number of significant digits over which the vortensity is represented, and many vortices absent from the double precision case.

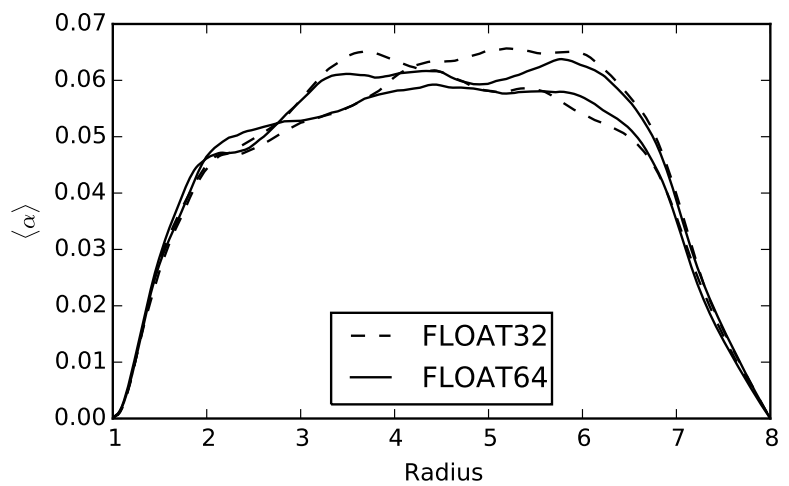

FIG. 21.- Value of $\alpha$ time averaged between 100 and 200 orbits at the disk inner edge, as a function of radius, for double precision calculations (solid lines) and for single precision calculations (dashed lines).

and their interactions with forming planets (Paardekooper \& Mellema 2008, Jang-Condell 2008; Lega et al. 2014). For this purpose we have implemented a simple radiative transfer module in FARGO3D, based on a gray approximation and flux limited diffusion, and a two-temperature (gas and photons) approach (Bitsch et al. 2013). Similarly, for the description of passive disks, the irradiation of the disk's photosphere by the stellar light is crucially important (Dullemond 2000; Dullemond et al. 2001; D'Alessio et al. 1998; Fung \& Artymowicz 2014), and we have implemented a module to account for this effect by ray tracing. Ray tracing requires radial integration of the zones optical depths, which can be tricky on parallel platforms, especially GPUs. We will present in a forthcoming work our implementation strategy for the radiative transfer and stellar irradiation modules.

The importance of using very large resolution for computations of embedded planets is becoming increasingly evident. Such resolution is way beyond current computational resources for global disk calculations with meshes with uniform resolution. A strategy can be to have smoothly variable cell sizes in order to achieve small cells in the planet vicinity (Bate et al. 2003). Another strategy can be the use of nested 
meshes of increasing resolution (D'Angelo et al.2003). This is the approach that we use in FARGO3D. There is an ongoing project aimed at merging the nested-mesh capability of the JUPITER code (Szulágyi et al.2014) with the FARGO3D code.

Other improvements have been the implementation of multi-fluid capability, and the incorporation of Hall effects and ambipolar diffusion to the MHD solver. Each of these new features will be presented in future publications.

Pablo Benítez-Llambay acknowledges financial support from CONICET and the computational resources provided by IATE and CCAD (Universidad Nacional de Córdoba). F. Masset acknowledges support from CONACyT grant 178377. We thank Sébastien Fromang for discussions and guidance during the development of the MHD solver, and for comments on an early version of this work. We thank Gloria Koenigsberger for a thorough reading of this manuscript, and constructive comments. We thank Ulises Amaya Olvera, Reyes García Carreón and Jérôme Verleyen for their assistance in setting up the GPU cluster on which most of the calculations presented here have been run.

\section{APPENDIX}

\section{GEOMETRIC COEFFICIENTS}

During a full update on the mesh, one requires at several places the volume of a zone, the surface of all its faces, and the length of its edges. In order to save memory (which is always a concern on GPUs) without sacrificing speed, we define a number of one-dimensional arrays, and use products of these arrays to obtain the face surfaces or the zone volumes. Our aim is that on relatively modest setups, these one-dimensional arrays may fit in the so-called constant memory, which is cached (fast) read-only memory on board the graphics card ${ }^{24}$. In FARGO3D the cell size is uniform in $X$ (hence azimuth in cylindrical or spherical geometries). This is a limitation imposed by the (nearly systematic) use of orbital advection along this direction ${ }^{25}$.

\section{Surfaces}

We have the relationships:

$$
\begin{aligned}
& S_{x}(j, k)=s_{x}^{j}(j) s_{x}^{k}(k) \\
& S_{y}(j, k)=s_{y}^{j}(j) s_{y}^{k}(k) \\
& S_{z}(j, k)=s_{z}^{j}(j) s_{z}^{k}(k),
\end{aligned}
$$

where the coefficients $s_{x / y / z}^{j / k}$ are defined in Tab. 5, and where $S_{A}(j, k)$ is the surface of the lower face of cell $(j, k)$ perpendicular to direction $A$ (the value of $i$ is unimportant for the reason given above, regardless of the geometry).

\section{Volumes}

As we more often use the inverse of the volume of a zone than its mere value, and since divisions have a high computational cost, we prefer to have an expression that directly gives us the inverse of a cell volume. We use:

$$
V^{-1}=\frac{V_{j}(j)^{-1}}{s_{y}^{k}(k)},
$$

where $V$ is the cell volume, and where the coefficients $V_{j}(j)^{-1}$ are defined in Tab. 5. We leave as an exercise to the reader to check that Eqs. A1 and (A4) lead to the exact surfaces and (inverse) volumes in all cases.

\section{VISCOUS STRESS TENSOR}

We provide hereafter the expression of its components in the three geometries (see e.g. Tassoul 1978), the different terms being written exactly as implemented ${ }^{26}$.

\section{Cartesian coordinates:}

$$
\tau_{i j}=-\rho v\left(\partial_{i} v_{j}+\partial_{j} v_{i}-\frac{2}{3} \delta_{i j} \nabla \cdot \vec{v}\right)
$$


FARGO3D

\begin{tabular}{|r|c|c|c|}
\hline factor & Cartesian & cylindrical & spherical \\
\hline$s_{x}^{j}(j)$ & $Y_{\max }-Y_{\min }$ & $Y_{\max }-Y_{\min }$ & $\frac{1}{2}\left(Y_{\max }^{2}-Y_{\min }^{2}\right)$ \\
\hline$s_{x}^{k}(k)$ & $Z_{\max }-Z_{\min }$ & $Z_{\max }-Z_{\min }$ & $Z_{\max }-Z_{\min }$ \\
\hline$s_{y}^{j}(j)$ & 1 & $Y_{\min } \Delta x$ & $Y_{\min }^{2} \Delta x$ \\
\hline$s_{y}^{k}(k)$ & $\Delta x\left(Z_{\max }-Z_{\min }\right)$ & $Z_{\max }-Z_{\min }$ & $\cos \left(Z_{\min }\right)-\cos \left(Z_{\max }\right)$ \\
\hline$s_{z}^{j}(j)$ & $\Delta x\left(Y_{\max }-Y_{\min }\right)$ & $\frac{1}{2} \Delta x\left(Y_{\max }^{2}-Y_{\min }^{2}\right)$ & $\frac{1}{2} \Delta x\left(Y_{\max }^{2}-Y_{\min }^{2}\right)$ \\
\hline$s_{z}^{k}(k)$ & 1 & 1 & $\sin \left(Z_{\min }\right)$ \\
\hline$V_{j}(j)^{-1}$ & $\left(Y_{\max }-Y_{\min }\right)^{-1}$ & $2 /\left[\left(Y_{\max }^{2}-Y_{\min }^{2}\right) \Delta x\right]$ & $3 /\left[\left(Y_{\max }^{3}-Y_{\min }^{3}\right) \Delta x\right]$ \\
\hline
\end{tabular}

TABLE 5

ONE DIMENSIONAL COEFFICIENTS IN THE THREE DIFFERENT GEOMETRIES. THE MEANING OF THE $X, Y$ AND $Z$ COORDINATES FOR EACH GEOMETRY IS GiVEN BY TAB. 1 IN EACH ROW, THE COEFFICIENT EITHER DEPENDS ON $j$ OR $k$, NEVER ON BOTH. THE MIN/MAX SUBSCRIPT REFERS TO THE LOCATION (IN $Y$ OR $Z$, DEPENDING ON THE CASE) OF THE LOWER/UPPER SIDE OF THE CELL (AT LOCATION $j, k$, THE VALUE OF INDEX $i$ BEING IRRELEVANT).

\section{Cylindrical coordinates::}

$$
\begin{aligned}
\tau_{\phi \phi} & =-\rho v\left[2\left(\frac{1}{r} \partial_{\phi} v_{\phi}+\frac{u_{r}}{r}\right)-\frac{2}{3} \nabla \cdot \vec{v}\right] \\
\tau_{r r} & =-\rho v\left(2 \partial_{r} v_{r}-\frac{2}{3} \nabla \cdot \vec{v}\right) \\
\tau_{z z} & =-\rho v\left(2 \partial_{z} v_{z}-\frac{2}{3} \nabla \cdot \vec{v}\right) \\
\tau_{\phi r}=\tau_{r \phi} & =-\rho v\left(\partial_{r} v_{\phi}-\frac{v_{\phi}}{r}+\frac{1}{r} \partial_{\phi} v_{r}\right) \\
\tau_{r z}=\tau_{z r} & =-\rho v\left(\partial_{r} v_{z}+\partial_{z} v_{r}\right) \\
\tau_{\phi z}=\tau_{z \phi} & =-\rho v\left(\partial_{z} v_{\phi}+\frac{1}{r} \partial_{\phi} v_{z}\right) .
\end{aligned}
$$

\section{Spherical coordinates::}

$$
\begin{aligned}
\tau_{r r} & =-\rho v\left(2 \partial_{r} v_{r}-\frac{2}{3} \nabla \cdot \vec{v}\right) \\
\tau_{\phi \phi} & =-\rho v\left[2\left(\frac{1}{r \sin \theta} \partial_{\phi} v_{\phi}+\frac{v_{r}}{r}+\frac{v_{\theta} \cot \theta}{r}\right)-\frac{2}{3} \nabla \cdot \vec{v}\right] \\
\tau_{\theta \theta} & =-\rho v\left[2\left(\frac{1}{r} \partial_{\theta} v_{\theta}+\frac{v_{r}}{r}\right)-\frac{2}{3} \nabla \cdot \vec{v}\right] \\
\tau_{\phi r}=\tau_{r \phi} & =-\rho v\left(\frac{1}{r \sin \theta} \partial_{\phi} v_{r}+\partial_{r} v_{\phi}-\frac{v_{\phi}}{r}\right) \\
\tau_{r \theta}=\tau_{\theta r} & =-\rho v\left(\partial_{r} v_{\theta}-\frac{v_{\theta}}{r}+\frac{1}{r} \partial_{\theta} v_{r}\right) \\
\tau_{\phi \theta}=\tau_{\theta \phi} & =-\rho v\left[\frac{\sin \theta}{r} \partial_{\theta}\left(\frac{v_{\phi}}{\sin \theta}\right)+\frac{1}{r \sin \theta} \partial_{\phi} v_{\theta}\right] .
\end{aligned}
$$

The different components of the stress tensor are located at different positions. The diagonal terms are zone centered, whereas the cross terms are defined at the middle of the edges. For instance, inside a given cell, $\tau_{x y}$ is defined at the middle of the edge along the $z$ dimension which has the lowest value for $x$ and $y$. By circular permutation of the indices, the centering of other components ensues. The position of the different components is depicted in Fig. 22.

The different components of the stress tensor, once calculated, are used to update directly the components of the velocity ${ }^{27}$. This corresponds to cell $7 \mathrm{~d}$ of Fig. 2. We list hereafter the partial derivative equations that correspond to this substep, in the three geometries.

\section{Cartesian coordinates:}

$$
\partial_{t} v_{i}=-\partial_{j} \tau_{i j}
$$




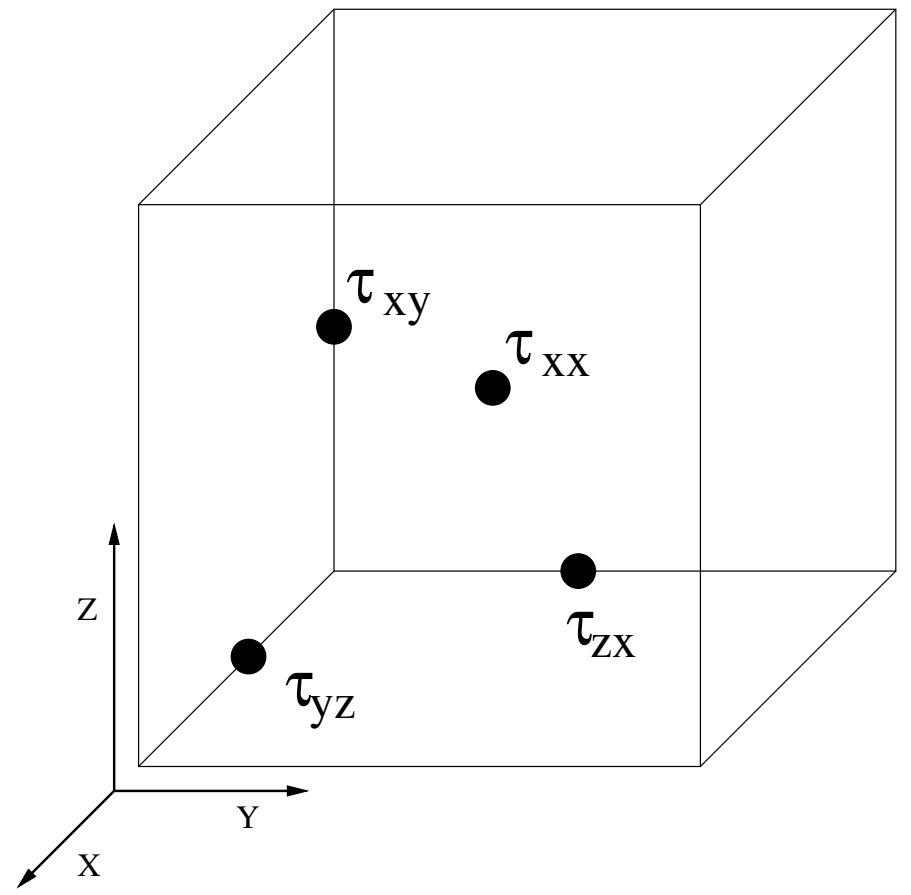

FIG. 22.- Location of the different components of the stress tensor.

\section{Cylindrical coordinates::}

$$
\begin{aligned}
& \partial_{t} v_{\phi}=-\frac{1}{\rho}\left[\frac{1}{r^{2}} \partial_{r}\left(r^{2} \tau_{\phi r}\right)+\frac{1}{r} \partial_{\phi} \tau_{\phi \phi}+\partial_{z} \tau_{\phi z}\right] \\
& \partial_{t} v_{r}=-\frac{1}{\rho}\left[\frac{1}{r} \partial_{r}\left(r \tau_{r r}\right)+\frac{1}{r} \partial_{\phi} \tau_{r \phi}-\frac{\tau_{\phi \phi}}{r}+\partial_{z} \tau_{r z}\right] \\
& \partial_{t} v_{z}=-\frac{1}{\rho}\left[\frac{1}{r} \partial_{r}\left(r \tau_{r z}\right)+\frac{1}{r} \partial_{\phi} \tau_{\phi z}+\partial_{z} \tau_{z z}\right] .
\end{aligned}
$$

\section{Spherical coordinates::}

$$
\begin{aligned}
\partial_{t} v_{\phi}= & -\frac{1}{\rho}\left[\frac{1}{r^{2}} \partial_{r}\left(r^{2} \tau_{\phi r}\right)+\frac{1}{r} \partial_{\theta} \tau_{\phi \theta}\right. \\
& \left.+\frac{1}{r \sin \theta} \partial_{\phi} \tau_{\phi \phi}+\frac{\tau_{r \phi}}{r}+\frac{2 \tau_{\theta \phi} \cot \theta}{r}\right] \\
\partial_{t} v_{r}= & -\frac{1}{\rho}\left[\frac{1}{r^{2}} \partial_{r}\left(r^{2} \tau_{r r}\right)+\frac{1}{r \sin \theta} \partial_{\theta}\left(\tau_{r \theta} \sin \theta\right)\right. \\
& \left.+\frac{1}{r \sin \theta} \partial_{\phi} \tau_{r \phi}-\frac{\tau_{\theta \theta}+\tau_{\phi \phi}}{r}\right] \\
\partial_{t} v_{\theta}= & -\frac{1}{\rho}\left[\frac{1}{r^{3}} \partial_{r}\left(r^{3} \tau_{r \theta}\right)+\frac{1}{r \sin \theta} \partial_{\theta}\left(\tau_{\theta \theta} \sin \theta\right)\right. \\
& \left.+\frac{1}{r \sin \theta} \partial_{\phi} \tau_{\theta \phi}-\frac{\tau_{\phi \phi} \cot \theta}{r}\right],
\end{aligned}
$$

where, again, the writing of the different terms reflects the actual implementation.

Notes

${ }^{1}$ Homepage: http://fargo.in2p3.fr

${ }^{2}$ This is activated by the use of the SHEARINGBOX macro-variable in the option (.opt) file.

${ }^{3}$ Note that owing to our convention that orbital advection is performed along the $X$ axis, the $X$ and $Y$ axis are swapped in our shearing sheet description with respect to standard notation. Also, either our $(X, Y, Z)$ base is no longer direct, or the rotation is directed toward decreasing $X$, which yields opposite sign for the Coriolis force.

${ }^{4}$ The source code corresponding to this step can be found in the files substep1_x.c, substep1_y.c and substep1_z.c. 
${ }^{5}$ The source code corresponding to this step can be found in the files substep2_a.c and substep2_b.c. The artificial viscous pressure is calculated in the first file, its gradient is then used in the second file. We had to split the operations to avoid race conditions, and to fully expose the parallelism of each substep. This kind of decomposition is common in our implementation.

${ }^{6}$ The source code corresponding to this step can be found in the file substep3.c.

${ }^{7}$ Source code in change_frame.c.

${ }^{8}$ The source code corresponding to the calculation of the slopes can be found in vanleer_x_a.c, vanleer_y_a.c and vanleer_z_a.c.

${ }^{9}$ The succession of operations involved in the PPA reconstruction is invoked in the file vanleerx_ppa.c, and the different operations called therein are found in the files fargo_ppa_a.c, fargo_ppa_b.c, fargo_ppa_c.c and fargo_ppa_d.c. Again, the operation is fragmented as necessary to avoid race conditions, thereby exposing the parallelism of each substep.

${ }^{10}$ Consistent transport is achieved by a division by the density of the quantities to transport, prior to perform the upwind evaluation. This division is carried out by invoking DivideByRho() in transport.c.

${ }^{11}$ The argument of the min function of Eq. 59 is evaluated in file cfl.c. Its minimum value is then sought in the file cfl_b.c, which also incorporates the shear time step limit of Eq. 773, when needed.

${ }^{12}$ The integer shift is implemented in file advect_shift.c, while the residual uniform shift uses the same function as standard advection, but with a different (uniform) velocity field. The invocation of the different sub steps is found in file transport.c. The user may switch between standard and orbital advection by commenting or commenting out the line FARGO_OPT += -DSTANDARD in the option (.opt) file.

${ }_{13}^{13}$ See the macrocommands $\mathrm{lxp}$ and $\mathrm{lxm}$ in define.h.

${ }^{14}$ The calculation of the star values is performed in file compute_star.c. The functions in this file admits an integer argument index which specifies whether the flow speed is to be included in the characteristics speed. It must be included for the calculation of the electric field in Eq. 84, whereas it must not be included for the evaluation of the Lorentz force in Eq. 86.

${ }^{15}$ Orbital advection is implemented in the file fargo mhd.c, which contains the invocation to the different sub steps, and in the file integrate_emf.c.

${ }^{16}$ the force exerted by the disk on the planets is evaluated in compute_force.c.

${ }^{17}$ This option is activated by the use of the compilation flag BM08.

${ }^{18}$ While some functions have been adapted from the former FARGO code, most of them have been written from scratch.

${ }^{19}$ Defined by the preprocessor variables NGHY and NGHZ in define.h.

${ }^{20}$ Through the use of the CreateField() and CreateFieldAlias() commands in LowTasks.c.

${ }^{21}$ In the files reduction_generic.c and reduction_generic_gpu.cu, in which the reduction operation is a macrocommand macro ( $\left.\mathrm{x}, \mathrm{y}\right)$, which can be arbitrary. These files perform the reduction in the $X$ direction, thereby returning an array of dimension $n-1$ for a run in $n$ dimensions. The final reduction of that intermediate array is always performed on the CPU. The generality of the generic reductions is exploited in files reduction_sum.c, reduction_min.c, reduction_min_device.cu and reduction_sum_device.cu.

${ }_{22}^{2}$ In addition to code execution timing, one can test the speed up on individual routines using the FARGO_SPEEDUP() macrocommand defined in define.h.

${ }^{23}$ By the use of the compilation option MPICUDA $=1$.

${ }^{24}$ The compilation variable BIGMEM controls this behavior: when set to $\Theta$ the constant memory is used, while when set to 1 the standard, global memory is used to store them.

${ }^{25}$ The zone width in $X$ is accessible throughout the code via the global variable Dx.

${ }^{26}$ Respectively in files visctensor_cart.c, visctensor_cyl.c and visctensor_sph.c.

${ }^{27}$ Respectively in files addviscosity_cart.c, addviscosity_cyl.c and addviscosity_sph.c.

\section{REFERENCES}

Balsara, D. S. \& Spicer, D. S. 1999a, Journal of Computational Physics, 153,671

Balsara, D. S. \& Spicer, D. S. 1999b, Journal of Computational Physics, 149,270

Baruteau, C., Fromang, S., Nelson, R. P., \& Masset, F. 2011, A\&A, 533, A84

Baruteau, C. \& Masset, F. 2008, ApJ, 678, 483

Bate, M. R., Lubow, S. H., Ogilvie, G. I., \& Miller, K. A. 2003, MNRAS, 341,213

Bitsch, B., Crida, A., Morbidelli, A., Kley, W., \& Dobbs-Dixon, I. 2013, A\&A, 549, A124

Brio, M. \& Wu, C. C. 1988, Journal of Computational Physics, 75, 400

Bryan, G. L., Norman, M. L., O’Shea, B. W., et al. 2014, ApJS, 211, 19

Cash, J. R. \& Karp, A. H. 1990, ACM Trans. Math. Softw., 16, 201

Casoli, J. \& Masset, F. S. 2009, ApJ, 703, 845

Colella, P. \& Woodward, P. R. 1984, Journal of Computational Physics, 54, 174

D’Alessio, P., Cantö, J., Calvet, N., \& Lizano, S. 1998, ApJ, 500, 411

D’Angelo, G., Kley, W., \& Henning, T. 2003, ApJ, 586, 540

de Val-Borro, M., Edgar, R. G., Artymowicz, P., et al. 2006, MNRAS, 695

Dullemond, C. P. 2000, A\&A, 361, L17

Dullemond, C. P., Dominik, C., \& Natta, A. 2001, ApJ, 560, 957

Duncan, M. J., Levison, H. F., \& Lee, M. H. 1998, AJ, 116, 2067

Evans, C. R. \& Hawley, J. F. 1988, ApJ, 332, 659

Fromang, S., Hennebelle, P., \& Teyssier, R. 2006, A\&A, 457, 371

Fung, J. \& Artymowicz, P. 2014, ApJ, 790, 78

Fung, J., Shi, J.-M., \& Chiang, E. 2014, ApJ, 782, 88

Gardiner, T. A. \& Stone, J. M. 2005, Journal of Computational Physics, 205, 509

Godunov, S. 1959, Matematicheskii Sbornik, 47

Harris, M. 2007, Optimizing parallel reduction in CUDA, Tech. rep., NVIDIA Corporation

Hawley, J. F. \& Stone, J. M. 1995, Computer Physics Communications, 89, 127

Jang-Condell, H. 2008, ApJ, 679, 797

Johnson, B. M., Guan, X., \& Gammie, C. F. 2008, ApJS, 177, 373
Kley, W. 1998, A\&A, 338, L37

Kley, W., Bitsch, B., \& Klahr, H. 2009, A\&A, 506, 971

Lega, E., Crida, A., Bitsch, B., \& Morbidelli, A. 2014, MNRAS, 440, 683

Li, H., Finn, J. M., Lovelace, R. V. E., \& Colgate, S. A. 2000, ApJ, 533, 1023

Lin, M.-K. \& Papaloizou, J. C. B. 2010, MNRAS, 405, 1473

Londrillo, P. \& Del Zanna, L. 2000, ApJ, 530, 508

Lovelace, R. V. E., Li, H., Colgate, S. A., \& Nelson, A. F. 1999, ApJ, 513, 805

Lynden-Bell, D. \& Pringle, J. E. 1974, MNRAS, 168, 603

Masset, F. 2000, A\&AS, 141, 165

Mignone, A., Flock, M., Stute, M., Kolb, S. M., \& Muscianisi, G. 2012,

A\&A, 545, A152

Morbidelli, A. \& Nesvorny, D. 2012, A\&A, 546, A18

Norman, M. L., Wilson, J. R., \& Barton, R. T. 1980, ApJ, 239, 968

Orszag, S. A. \& Tang, C.-M. 1979, Journal of Fluid Mechanics, 90, 129

Paardekooper, S.-J. \& Mellema, G. 2008, A\&A, 478, 245

Robertson, B. E., Kravtsov, A. V., Gnedin, N. Y., Abel, T., \& Rudd, D. H.

2010, Mon.Not.Roy.Astron.Soc., 401, 2463

Ryu, D., Ostriker, J. P., Kang, H., \& Cen, R. 1993, ApJ, 414, 1

Schive, H.-Y., Tsai, Y.-C., \& Chiueh, T. 2010, ApJS, 186, 457

Schneider, E. E. \& Robertson, B. E. 2015, ApJS, 217, 24

Sorathia, K. A., Reynolds, C. S., Stone, J. M., \& Beckwith, K. 2012, ApJ,

749,189

Speith, R. \& Riffert, H. 1999, Journal of Computational and Applied

Mathematics, 109, 231

Stone, J. M. \& Gardiner, T. A. 2010, ApJS, 189, 142

Stone, J. M. \& Norman, M. L. 1992a, ApJS, 80, 753

Stone, J. M. \& Norman, M. L. 1992b, ApJS, 80, 791

Szulágyi, J., Morbidelli, A., Crida, A., \& Masset, F. 2014, ApJ, 782, 65

Tassoul, J.-L. 1978, Theory of rotating stars (Princeton University Press)

Trac, H. \& Pen, U.-L. 2004, 9, 443

van Leer, B. 1977, Journal of Computational Physics, 23, 276 